\title{
INSTITUTIONAL INVESTORS AND STOCK MARKET VOLATILITY*
}

\author{
XAVier Gabaix \\ Parameswaran Gopikrishnan \\ VASILIKI Plerou \\ H. Eugene Stanley
}

\begin{abstract}
We present a theory of excess stock market volatility, in which market movements are due to trades by very large institutional investors in relatively illiquid markets. Such trades generate significant spikes in returns and volume, even in the absence of important news about fundamentals. We derive the optimal trading behavior of these investors, which allows us to provide a unified explanation for apparently disconnected empirical regularities in returns, trading volume and investor size.
\end{abstract}

\section{INTRODUCTION}

Ever since Shiller [1981], economists have sought to understand the origins of volatility in stock market prices, which appears to exceed the predictions of simple models with rational expectations and constant discounting. ${ }^{1}$ Even after the fact, it is hard to explain changes in the stock market using only observable news [Cutler, Poterba, and Summers 1989; Fair 2002; Roll 1988].

We present a model in which volatility is caused by the trades of large institutions. Institutional investors appear to be important for the low-frequency movements of equity prices, as

* We thank our editor and referees for very helpful comments. We also thank Tobias Adrian, Nicholas Barberis, Olivier Blanchard, Jean-Philippe Bouchaud, John Campbell, Victor Chernozhukov, Emanuel Derman, Peter Diamond, Alexander Edmans, Edward Glaeser, Joel Hasbrouck, David Hirshleifer, Harrison Hong, Soeren Hvidjkaer, Ivana Komunjer, David Laibson, Augustin Landier, Ananth Madhavan, Andrew Metrick, Lasse Pedersen, Thomas Philippon, Marc Potters, Jonathan Reuter, Bryan Routledge, Gideon Saar, Andrei Shleifer, Didier Sornette, Dimitri Vayanos, Jessica Wachter, Jiang Wang, Jeffrey Wurgler, and seminar participants at University of California, Berkeley, Département et Laboratoire d'Economie Théorique et Appliquée, Duke University, Harvard University, Johns Hopkins University, Kellogg School of Management, Massachusetts Institute of Technology, National Bureau of Economic Research, New York University, Princeton University, Stanford University, Wharton School of Business, the Econophysics conferences in Indonesia and Japan, the Western Finance Association, and Econometric Society meetings. We thank Fernando Duarte, Tal Fishman, and David Kang for their research assistance. Gopikrishnan's contribution was part of his Ph.D. thesis. We thank Morgan Stanley, the National Science Foundation, and the Russell Sage Foundation for support.

1. See also Campbell and Shiller [1988], French and Roll [1986], and LeRoy and Porter [1981].

๑ 2006 by the President and Fellows of Harvard College and the Massachusetts Institute of Technology.

The Quarterly Journal of Economics, May 2006 
shown by Gompers and Metrick [2001]. Understanding better the behavior of institutional investors also sheds light on many issues, such as momentum and positive feedback trading [Chae and Lewellen 2005; Cohen, Gompers, and Vuolteenaho 2002; Choe, Kho, and Stulz 1999; Hvidkjaer 2005], bubbles [Brunnermeier and Nagel 2004], liquidity provision [Campbell, Ramadorai, and Vuolteenaho 2005], and the importance of indexing [Goetzman and Massa 2003]. We further this research by analyzing how trading by individual large investors may create price movements that are hard to explain by fundamental news.

In our theory, spikes in trading volume and returns are created by a combination of news and the trades by large investors. Suppose that news or proprietary analysis induces a large investor to trade a particular stock. Since his desired trading volume is then a significant proportion of daily turnover, he will moderate his actual trading volume to avoid paying too much in price impact. ${ }^{2}$ The optimal volume will nonetheless remain large enough to induce a significant price change.

Traditional measures, such as variances and correlations, are of limited use in analyzing spikes in market activity. Many empirical moments are infinite; moreover, their theoretical analysis is typically untractable. ${ }^{3}$ Instead, a natural object of analysis turns out to be the tail exponent of the distribution, for which some convenient analytical techniques apply. Furthermore, there is much empirical evidence on the tails of the distributions, which appear to be well approximated by power laws. For example, the distribution of returns $r$ over daily or weekly horizons decays according to $P(|r|>x) \sim x^{-\zeta_{r}}$, where $\zeta_{r}$ is the tail or Pareto exponent. ${ }^{4}$ This accumulated evidence on tail behavior is useful to guide and constrain any theory of the impact of large investors. Specifically, our theory unifies the following stylized facts.

(i) the power law distribution of returns, with exponent $\zeta_{r} \simeq 3$

(ii) the power law distribution of trading volume, with exponent $\zeta_{q} \simeq 1.5$

(iii) the power law of price impact;

(iv) the power law distribution of the size of large investors, with exponent $\zeta_{S} \simeq 1$.

2. See subsection II.C.

3. The variance of volume and the kurtosis of returns are infinite. Section II provides more details.

4. Appendix 1 reviews the relevant techniques. 
Existing models have difficulty in explaining facts (i)-(iv) together, not only the power law behavior in general, but also the specific exponents. For example, efficient markets theories rely on news to move stock prices and thus can explain the empirical finding only if news is power law distributed with an exponent $\zeta_{r} \simeq 3$. However, there is nothing a priori in the efficient markets hypothesis that justifies this assumption. Similarly, GARCH models generate power laws, but need to be fine-tuned to replicate the exponent of $3 .^{5}$

We rely on previous research to explain (iv), and develop a trading model to explain (iii). We use these facts together to derive the optimal trading behavior of large institutions in relatively illiquid markets. The fat-tailed distribution of investor sizes generates a fat-tailed distribution of volumes and returns. When we derive the optimal trading behavior of large institutions, we are able to replicate the specific values for the power law exponents found in stylized facts (i) and (ii). ${ }^{6}$

In addition to explaining the above facts, an analysis of tail behavior may have a number of wider applications in option pricing, ${ }^{7}$ risk management, and the debate on the importance of large returns for the equity premium [Barro 2006; Rietz 1988; Routledge and Zin 2004; Weitzman 2005].

Our paper draws on several literatures. The behavioral finance literature [Barberis and Thaler 2003; Hirshleifer 2001; Shleifer 2000] describes mechanisms by which large returns obtain without significant changes in fundamentals. We propose that these extreme returns often result from large idiosyncratic trades of institutions. The microstructure literature [Biais, Glosten, and Spatt 2005; O'Hara 1995] shows that order flow can explain a large fraction of exchange rate movements [Evans and Lyons 2002] and stock price movements, including the covariance between stocks [Hasbrouck and Seppi 2001]. Previous papers combine these behavioral, microstructure and asset pricing elements to explain the impact of limited liquidity and demand

5. Also, GARCH models are silent about the economic origins of the tails, and about trading volume.

6. This includes the relative fatness documented by facts (i), (ii), and (iv) (note that a higher exponent means a thinner tail). Since large traders moderate their trading volumes, the distribution of volumes is less fat-tailed than that of investor sizes. In turn, a concave price impact function leads to return distributions being less fat-tailed than volume distributions.

7. Our theory indicates that trading volume should help forecast the probability of large returns. Marsh and Wagner [2004] provides evidence consistent with that view. 
pressures on asset prices [Acharya and Pedersen 2005; Gompers and Metrick 2001; Pritsker 2005; Shleifer 1986; Wurgler and Zhuravskaya 2002]. We complement this research by focusing on tail behavior, partially in the hope that understanding extreme events allows us to understand standard market behavior better.

This article is also part of a broader movement utilizing concepts and methods from physics to study economic issues, a literature sometimes referred to as "econophysics." ${ }^{8}$ Econophysics is similar in spirit to behavioral economics in that it postulates simple plausible rules of agent behavior, and explores their implications. However, it differs by putting less emphasis on the psychological microfoundations, and more on the results of the interactions among agents.

Section II presents stylized facts on the tail behavior of financial variables. Section III then contains our baseline model that connects together power laws. Section IV discusses various extensions. Section V concludes. Appendix 1 is a primer on power law mathematics.

\section{The Empirical Findings That Motivate Our Theory}

This section presents the empirical facts that motivate our theory, and provides a self-contained tour of the empirical literature on power laws.

\section{II.A. The Power Law Distribution of Price Fluctuations: $\zeta_{r} \simeq 3$}

The tail distribution of returns has been analyzed in a series of studies that uses an ever increasing number of data points [Jansen and de Vries 1991; Lux 1996; Gopikrishnan, Plerou, Amaral, Meyer, and Stanley 1999; Plerou, Gopikrishnan, Amaral, Meyer, and Stanley 1999]. Let $r_{t}$ denote the logarithmic return over a time interval $\Delta t$. The distribution function of returns for the 1000 largest U. S. stocks and several major international indices has been found to be ${ }^{9}$

8. Antecedents are Simon [1955] and Mandelbrot [1963]. More recent research includes Bak, Chen, Scheinkman, and Woodford [1993], Bouchaud and Potters [2003], Gabaix [1999, 2005], Plerou, Gopikrishnan, Amaral, Meyer, and Stanley [1999], Levy, Levy, and Solomon [2000], Lux and Sornette [2002], and Mantegna and Stanley [1995, 2000]. See also Arthur et al. [1997], Blume and Durlauf [2005], Brock and Hommes [1998], Durlauf [1993], and Jackson and Rogers [2005] for work in a related vein.

9. To compare quantities across different stocks, we normalize variables such as $r$ and $q$ by the second moments if they exist, otherwise by the first moments. 


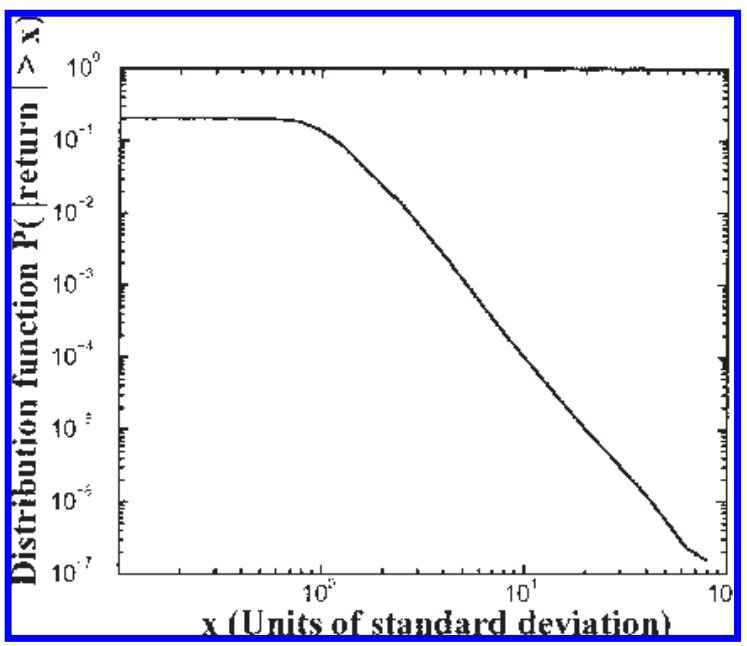

Figure I

Empirical cumulative distribution of the absolute values of the normalized fifteen-minute returns of the 1000 largest companies in the Trades And Quotes database for the two-year period 1994-1995 (12 million observations). We normalize the returns of each stock so that the normalized returns have a mean of 0 and a standard deviation of 1 . For instance, for a stock $i$, we consider the returns $r_{i t}^{\prime}=\left(r_{i t}-r_{i}\right) / \sigma_{r, i}$, where $r_{i}$ is the mean of the $r_{i t}$ 's and $\sigma_{r, i}$ is their standard deviation. In the region $2 \leq x \leq 80$ we find an ordinary least squares fit $\ln P(|r|>x)=-\zeta_{r} \ln x+b$, with $\zeta_{r}=3.1 \pm 0.1$. This means that returns are distributed with a power law $P(|r|>x) \sim x^{-\zeta_{r}}$ for $x$ between 2 and 80 standard deviations of returns. Source: Gabaix et al. [2003].

$$
P(|r|>x) \sim 1 / x^{\zeta_{r}} \text { with } \zeta_{r} \simeq 3
$$

Here, $\sim$ denotes asymptotic equality up to numerical constants. ${ }^{10}$ This relationship holds for positive and negative returns separately and is best illustrated in Figure I. It plots the cumulative probability distribution of the population of normalized absolute returns, with $\ln x$ on the horizontal axis and $\ln P(|r|>x)$ on the vertical axis. It shows that

$$
\ln P(|r|>x)=-\zeta_{r} \ln x+\text { constant }
$$

For instance, for a stock $i$, we consider the returns $r_{i t}^{\prime}=\left(r_{i t}-r_{i}\right) / \sigma_{r, i}$, where $r_{i}$ is the mean of the $r_{i t}$ and $\sigma_{r, i}$ is their standard deviation. For volume, which has an infinite standard deviation, we use the normalization $q_{i t}^{\prime}=q_{i t} / q_{i}$, where $q_{i t}$ is the raw volume, and $q_{i}$ is the absolute deviation: $q_{i}=\overline{\left|q_{i t}-q_{i t}\right|}$.

10. Formally, $f(x) \sim g(x)$ means that $f(x) / g(x)$ tends toward a positive constant (not necessarily 1 ) as $x \rightarrow \infty$. 


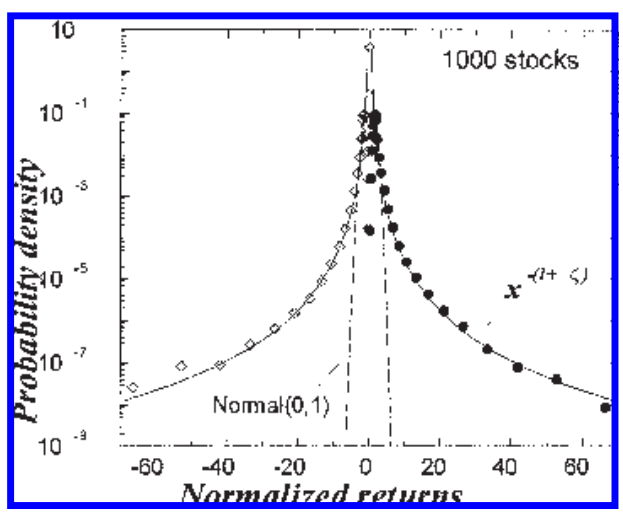

FiguRE II

Probability density function of the returns normalized five minute returns of the 1000 largest companies in the Trades And Quotes database for the two-year period 1994-1995. The values in the center of the distribution arise from the discreteness in stock prices, which are set in units of fractions of U. S. dollars, usually $1 / 8,1 / 16$, or $1 / 32$. The solid curve is a power-law fit in the region $2 \leq x \leq 80$. We find that $\zeta=3.1 \pm 0.03$ for the positive tail, and $\zeta=2.84 \pm 0.12$ for the negative tail. The dotted line represents a Gaussian density. Source: Plerou et al. [1999].

yields a good fit for $|r|$ between 2 and 80 standard deviations. OLS estimation yields $-\zeta_{r}=-3.1 \pm 0.1$, i.e., (1). It is not automatic that this graph should be a straight line, or that the slope should be -3 : in a Gaussian world it would be a concave parabola. In the following, we shall refer to equation (1) as "the cubic law of returns." 11

Furthermore, the 1929 and 1987 "crashes" do not appear to be outliers to the power law distribution of daily returns [Gabaix, Gopikrishnan, Plerou, and Stanley 2005]. Thus, there may not be a need for a special theory of "crashes": extreme realizations are fully consistent with a fat-tailed distribution. ${ }^{12}$

Equation (1) appears to hold internationally [Gopikrishnan, Plerou, Amaral, Meyer, and Stanley 1999]. For example, Figure

11. The particular value $\zeta_{r} \simeq 3$ is consistent with a finite variance, but moments higher than 3 are unbounded. $\zeta_{r} \simeq 3$ contradicts the "stable Paretian hypothesis" of Mandelbrot [1963], which proposes that financial returns follow a Lévy stable distribution. A Lévy distribution has an exponent $\zeta_{r} \leq 2$, which is consistent with the empirical evidence [Fama 1963; McCulloch 1996; Rachev and Mittnick 2000].

12. Subsection IV.D reports quotes from the Brady report, which repeatedly marvels at how concentrated trading was on Monday, October 19, 1987. 


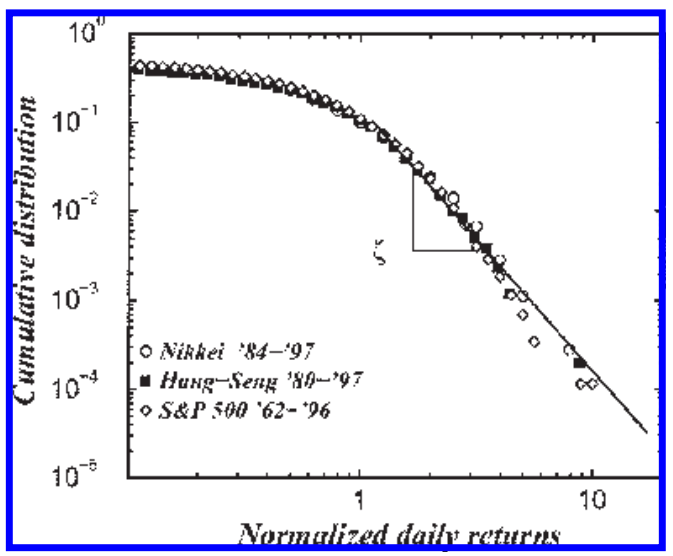

FIGURE III

Empirical cumulative distribution function of the absolute value of the daily return of the Nikkei (1984-1997), the Hang-Seng (1980-1997), and the S\&P 500 (1962-1996). The apparent power-law behavior in the tails is characterized by the exponents $\zeta_{r}=3.05 \pm 0.16$ (Nikkei), $\zeta_{r}=3.03 \pm 0.16$ (Hang-Seng), and $\zeta_{r}=$ $3.34 \pm 0.12(\mathrm{~S} \& \mathrm{P} 500)$. The fits are performed in the region $|r|$ between one and ten standard deviations of returns. Source: Gopikrishnan et al. [1999].

III shows that the distribution of returns for three different country indices are very similar. ${ }^{13}$

Having checked the robustness of the $\zeta_{r} \simeq 3$ finding across different stock markets, Plerou, Gopikrishnan, Amaral, Meyer, and Stanley [1999] examine firms of different sizes. ${ }^{14}$ Small firms have higher volatility than large firms, as is verified in Figure IVa. Moreover, the same diagram also shows similar slopes for the graphs of all four distributions. ${ }^{15}$ Figure IVb normalizes the

13. The empirical literature has proposed other distributions. We are more confident about our findings as they rely on a much larger number of data points, and hence quantify the tails more reliably. We can also explain previous findings in light of ours. Andersen, Bollerslev, Diebold, and Ebens [2001] show that the bulk of the distribution of realized volatility is lognormal. In independent work, Liu, Gopikrishnan, Cizeau, Meyer, Peng, and Stanley [1999] show that while this is true, the tails seem to be power law.

14. Some studies quantify the power-law exponent of foreign exchange fluctuations. The most comprehensive is probably Guillaume, Dacorogna, Davé, Müller, Olsen, and Pictet [1997], who calculate the exponent $\zeta_{r}$ of the price movements between the major currencies. At the shortest frequency $\Delta t=10$ minutes, they find exponents with average $\overline{\zeta_{r}}=3.44$, and a standard deviation 0.30 . This is tantalizingly close to the stock market findings, though the standard error is too high to draw sharp conclusions.

15. There is some dispersion in the measured exponent across individual stocks [Plerou, Gopikrishnan, Amaral, Meyer, and Stanley 1999]. This is expected, as least because measured exponents are noisy. Proposition 5 makes predictions about the determinants of a possible heterogeneity in the exponents. 


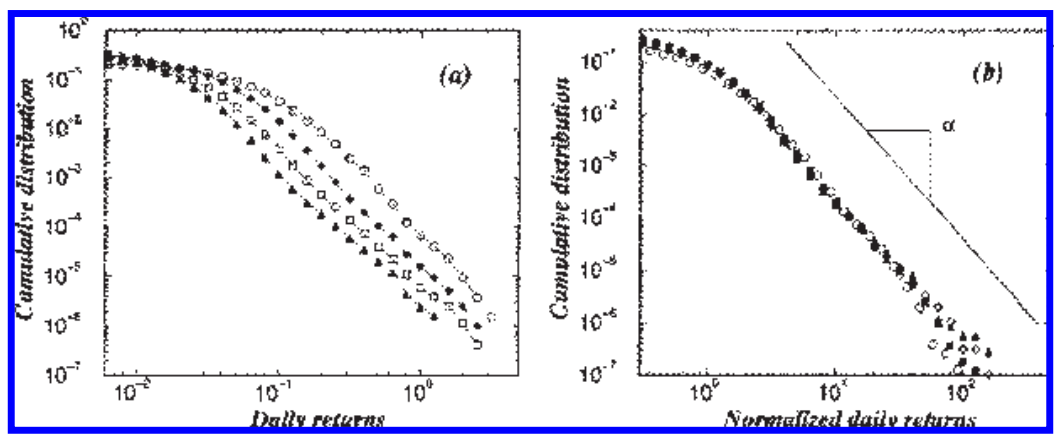

FiguRE IV

Cumulative distribution of the conditional probability $P(|r|>x)$ of the daily returns of companies in the CRSP database, 1962-1998. We consider the starting values of market capitalization $K$, define uniformly spaced bins on a logarithmic scale, and show the distribution of returns for each bin: $K \in\left[10^{5}, 10^{6}\right](\bigcirc), K \in$ $\left[10^{6}, 10^{7}\right](\bullet), K \in\left[10^{7}, 10^{8}\right](\square), K \in\left[10^{8}, 10^{9}\right](\mathbf{\Delta}) . K$ is measured in 1962 constant dollars. (a) Unnormalized returns. Each cumulative distribution corresponds to a bin of sizes. Small stocks are to the right, because they are more volatile. (b) Returns normalized by the average volatility $\sigma_{K}$ of each bin. The plots collapsed to an identical distribution, with $\zeta_{r}=2.70 \pm .10$ for the negative tail, and $\zeta_{r}=2.96 \pm .09$ for the positive tail. The horizontal axis displays returns that are as high as 100 standard deviations. Source: Plerou et al. [1999].

distribution of each size quantile by its standard deviation, so that the normalized distributions all have a standard deviation of 1 . The plots collapse on the same curve, and all have exponents close to $\zeta_{r} \simeq 3$.

The above results hold for relatively short time horizons-a day or less. ${ }^{16}$ Longer-horizon return distributions are shaped by two opposite forces. One force is that a finite sum of independent power law distributed variables with exponent $\zeta$ is also power-law distributed, with the same exponent $\zeta .{ }^{17}$ Thus, one expects the tails of monthly and even quarterly returns to remain power-law distributed. The second force is the central limit theorem, which says that if $T$ returns are aggregated, the bulk of the distribution converges to Gaussian. In sum, as we aggregate over $T$ returns, the central part becomes more Gaussian, while the tails remain a

16. Our analysis does not require exact power laws. It is enough that an important part of the tail distribution is well approximated by a power law. For instance, lognormal distributions with high variance are often well approximated by Pareto distributions. The exponent is then interpreted as a local exponent, i.e., $\zeta(x)=-x p^{\prime}(x) / p(x)-1$, rather than a global exponent.

17. This is one of the aggregation properties of power laws reviewed in Appendix 1. 
power law with exponent $\zeta$, but have an ever smaller probability, so that they may not even be detectable in practice. See Bouchaud and Potters [2003, pp. 33-35] for an example. In practice, the convergence to the Gaussian is slower than if returns were independently and identically distributed (i.i.d.), and one still sees fat tails at yearly horizons [Plerou, Gopikrishnan, Amaral, Meyer, and Stanley 1999, Figure 9]. A likely explanation is the autocorrelation of volatility and trading activity [Plerou, Gopikrishnan, Amaral, Gabaix, and Stanley 2000]. ${ }^{18}$ A useful extension of the present model would allow the desire to trade (or signal occurrences) to be autocorrelated, and might generate the right calibration of autocorrelation of volatility and slow convergence to a Gaussian.

In conclusion, the existing literature shows that while high frequencies offer the best statistical resolution to investigate the tails, power laws still appear relevant for the tails of returns at longer horizons, such as a month or even a year. ${ }^{19}$

\section{II.B. The Power Law Distribution of Trading Volume: $\zeta_{q} \simeq 3 / 2$}

To better constrain a theory of large returns, it is helpful to understand the structure of large trading volumes. Gopikrishnan, Plerou, Gabaix, and Stanley [2000] find that the size of individual trades for the 1000 largest U. S. stocks are also powerlaw distributed: ${ }^{20}$

$$
P(q>x) \sim 1 / x^{\zeta_{q}} \text { with } \zeta_{q} \simeq 3 / 2 .
$$

The precise value estimated is $\zeta_{q}=1.53 \pm .07$. Figure V illustrates: the density satisfies $p(q) \sim q^{-2.5}$, i.e., (3). The exponent of the distribution of individual trades is close to 1.5. Maslov and Mills [2001] likewise find $\zeta_{q}=1.4 \pm 0.1$ for the volume of individual market orders.

To test the robustness of this result, we examine 30 large stocks of the Paris Bourse from 1995-1999, which contain approximately 35 million records, and 250 stocks of the London Stock Exchange in 2001. As shown in Figure V, we find that $\zeta_{q}=$

18. Aggregation issues may also be important to understand the dispersion of exponents [Plerou, Gopikrishnan, Amaral, and Stanley 1999].

19. Dembo, Deuschel, and Duffie [2004], Ibragimov [2005], and Kou and Kou [2004] develop further the importance of fat tails in finance.

20 . We define volume as the number of shares traded. The dollar value traded yields very similar results, since, for a given security, it is essentially proportional to the number of shares traded. 


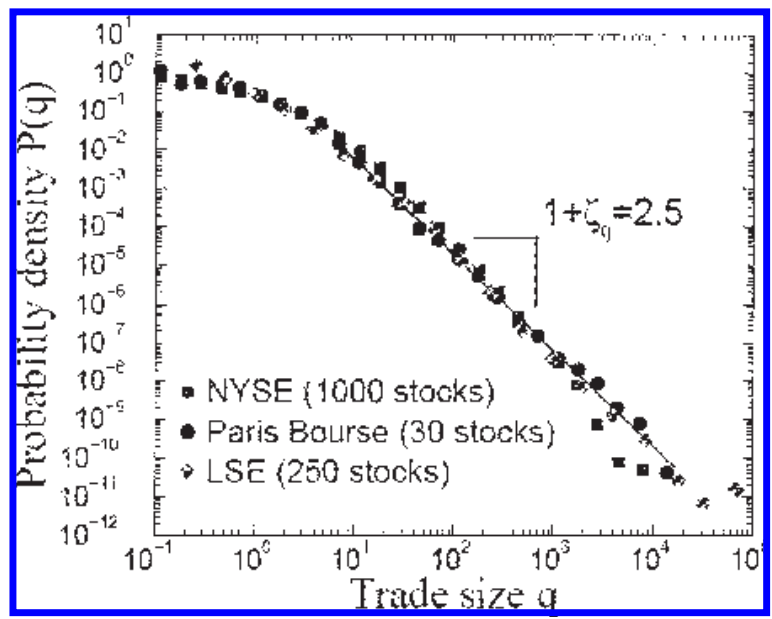

FiguRe V

Probability density of normalized individual transaction sizes $q$ for three stock markets: (i) NYSE for 1994-1995, (ii) the London Stock Exchange for 2001, and (iii) the Paris Bourse for 1995-1999. OLS fit yields $\ln p(x)=-\left(1+\zeta_{q}\right) \ln x+$ constant for $\zeta_{q}=1.5 \pm 0.1$. This means a probability density function $p(x) \sim$ $x^{-(1+\zeta q)}$, and a countercumulative distribution function $P(q>x) \sim x^{-\zeta q}$. The three stock markets appear to have a common distribution of volume, with a power-law exponent of $1.5 \pm 0.1$. The horizontal axis shows individual volumes that are up to $10^{4}$ times larger than the absolute deviation, $|q-\bar{q}|$.

$1.5 \pm 0.1$ for each of the three stock markets. The exponent appears essentially identical in the three stock markets, which is suggestive of universality.

The low exponent $\zeta_{q} \simeq 3 / 2$ indicates that the distribution of volumes is very fat tailed, and trading is very concentrated. Indeed, the 1 percent largest trades represent 28.5 percent $( \pm 0.6$ percent) of the total volume traded. ${ }^{21}$

The power law of individual trades continues to hold for volumes that are aggregated (for a given stock) at the horizon $\Delta t=15$ minutes [Gopikrishnan, Plerou, Gabaix, and Stanley 2000]:

$$
P(Q>x) \sim 1 / x^{\zeta Q} \text { with } \zeta_{Q} \simeq 3 / 2 .
$$

We refer to equations (3)-(4) as the "half-cubic law of trading volume."

21. The 0.1 percent largest trades represent $9.6 \pm 0.3$ percent of the total volume traded. We computed the statistics on the 100 largest stocks of the Trades And Quotes database in the period 1994-1995. 
It is intriguing that the exponent of returns should be 3 and the exponent of volumes should be 1.5. To see whether there is an economic connection between those values, we turn to the relation between return and volume.

\section{II.C. The Power Law of Price Impact: $r \sim V^{\gamma}$}

The microstructure literature generally confirms that substantial trades can have a large impact. Chan and Lakonishok [1993, 1995] estimate a range of 0.3 to 1 percent; Keim and Madhavan [1996] find 4 percent for smaller stocks. There are also many anecdotal examples of large investors affecting prices: see Brady [1988], Corsetti, Pesenti, and Roubini [2002], and Coyne and Witter [2002]. ${ }^{22}$

A simple calculation illustrates why one can expect that a large fund can move the market significantly. The typical yearly turnover of a stock is 50 percent of the shares outstanding [Lo and Wang 2001]: hence daily turnover is $0.5 / 250=0.2$ percent based on 250 trading days per year. Consider a moderately large fund, e.g., the thirtieth largest fund. At the end of 2000 , such a fund held 0.1 percent of the market and hence, on average, 0.1 percent of the capitalization of a given stock. ${ }^{23}$ To sell its entire holding, the fund will have to absorb $0.1 / 0.2$ or half of the daily turnover. This supports the idea that large funds are indeed large compared to the liquidity of the market, and that price impact will therefore be an important consideration.

We next present evidence that the price impact $r$ of a trade of size $V$ scales as

$$
r \sim k V^{\gamma},
$$

with $k>0,0 \leq \gamma \leq 1$, which yields a concave price impact function [Hasbrouck 1991; Hasbrouck and Seppi 2001; Plerou, Gopikrishnan, Gabaix, and Stanley 2002]. The parameterization $\gamma=1 / 2$ is often used, e.g., by Barra [1997], Gabaix, Gopikrishnan, Plerou, and Stanley [2003], Grinold and Kahn [1999], and Hasbrouck and Seppi [2001].

Equation (5) implies that $\zeta_{r}=\zeta_{V} / \gamma$ by rule (42) in Appendix

22. See also Chiyachantana, Jain, Jiang, and Wood [2004] for international evidence, and Jones and Lipson [2001] and Werner [2003] for recent U. S. evidence.

23. It had $\$ 19$ billion in assets under management. The total market capitalization of the New York Stock Exchange, the Nasdaq, and the American Stock Exchange was $\$ 18$ trillion. 
1. Hence, given $\zeta_{r}=3$ and $\zeta_{V}=3 / 2$, the value $\gamma=1 / 2$ is a particularly plausible null hypothesis. From this relationship, we see a natural connection between the power laws of returns and volumes.

The exact value of $\gamma$ is a topic of active research. We report here evidence on the null hypothesis $\gamma=1 / 2$. We start from the benchmark where, in a given time interval, $n$ blocks are traded, with volumes $V_{1}, \ldots, V_{n}$, of independent signs $\varepsilon_{i}= \pm 1$ with equal probability. Aggregate volume is $Q=\sum_{i=1}^{n} V_{i}$, and aggregate return is

$$
r=u+k \sum_{i=1}^{n} \varepsilon_{i} V_{i}^{1 / 2},
$$

where $u$ is some other orthogonal source of price movement. ${ }^{24}$ Then,

$$
\begin{aligned}
E\left[r^{2} \mid Q\right] & =\sigma_{u}^{2}+k^{2} E\left[\sum_{i} V_{i}+\sum_{i \neq j} \varepsilon_{i} \varepsilon_{i} V_{i}^{1 / 2} V_{j}^{1 / 2} \mid Q=\sum_{i} V_{i}\right] \\
& =\sigma_{u}^{2}+k^{2} Q+0 \\
E\left[r^{2} \mid Q\right] & =\sigma_{u}^{2}+k^{2} Q .
\end{aligned}
$$

Our results of Figure VI reveals an affine relation predicted by equation (7) for large volumes $Q$, rather than any clear sign of concavity or convexity. A formal test that we detail in Appendix 3 confirms this relation.

Measuring price impact and its dependence on order size is a complex problem due to the following reasons. First, order flow and returns are jointly endogenous. To our knowledge, virtually all empirical studies including ours, suffer from this lack of exogeneity in order flow.

Second, the unsplit size of orders is unobservable in most liquid markets. One observes the size of individual trades $q$, not the size of the desired block $V$. If one does not pay attention to aggregation, different exponents of price impact are measured,

24. Via equation (6), a model such as ours provides a foundation for stochastic clock representations of the type proposed by Clark [1973].

25. An exception is Loeb [1983], who collected bids on different size blocks of stock. Barra [1997] and Grinold and Kahn [1999, p. 453] report that the best fit of the Loeb data is a square root price impact. 


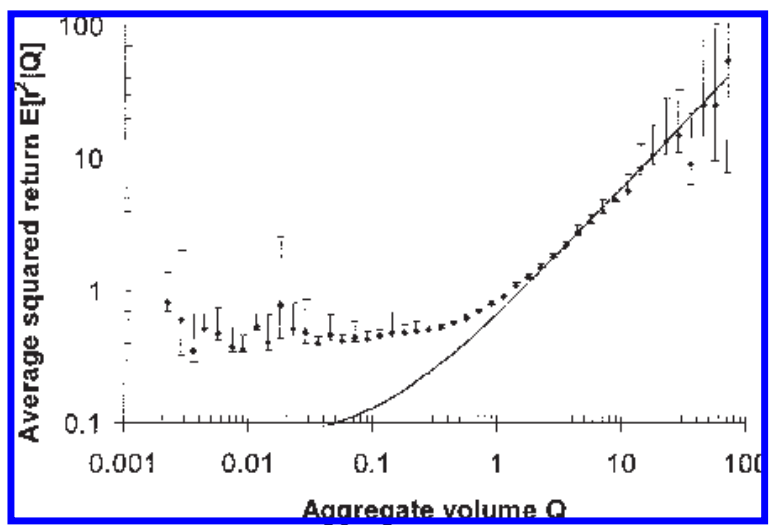

FiguRE VI

Conditional expectation $E\left[r^{2} \mid Q\right]$ of the squared return $r^{2}$ in $\Delta t=15$ minutes, given the aggregate volume $Q$ in $\Delta t . r$ is in units of standard deviation, and $Q$ in units of absolute deviation, $|Q-\bar{Q}|$. The results are averaged over the largest 100 stocks in the New York Stock Exchange market capitalization on January 1, 1994. The data span the two-year period 1994-1995 and are obtained from the Trades And Quotes database, which records all transactions for all listed securities in the NYSE, AMEX, and NASDAQ. Formal tests reported in Appendix 3 show that one cannot reject $E\left[r^{2} \mid Q\right]=\alpha+\beta Q$ large enough $(Q \geq 3)$. This is consistent with a square root price impact of large trades. Appendix 3 reports the procedure used to compute the 95 percent confidence intervals.

depending on the time horizon chosen [Plerou, Gopikrishnan, Gabaix, and Stanley 2002, 2004; Farmer and Lillo 2004]. ${ }^{26}$

Third, order flow is autocorrelated [Froot, O'Connell, and Seasholes 2001; Bouchaud, Gefen, Potters, and Wyart 2004; Lillo and Farmer 2004]. This autocorrelation could come from the actions of different traders. It is also predicted by models of optimal execution of trades [Almgren and Chriss 2000; Berstimas and Lo 1998; Gabaix, Gopikrishnan, Plerou, and Stanley 2003], as large transactions are split into smaller pieces. ${ }^{27}$

Although the empirical evidence we gathered is suggestive, measuring the curvature $\gamma$ of price impact more accurately will require better data and a technique to address the endogeneity of order flow. In particular, it would require knowing desired trading volumes, magnitude of price impact, and split of trades for a

26. In a related way, part of the linearity of equation (7) can arise because in some simple models total volume and squared returns depend linearly on the number of trades [Plerou, Gopikrishnan, Amaral, Gabaix, and Stanley 2000].

27. If the trades are executed in the same time window, equation (7) still holds. If they do not, the estimate of $\gamma$ is typically biased downward [Plerou, Gopikrishnan, Gabaix, and Stanley 2004]. 


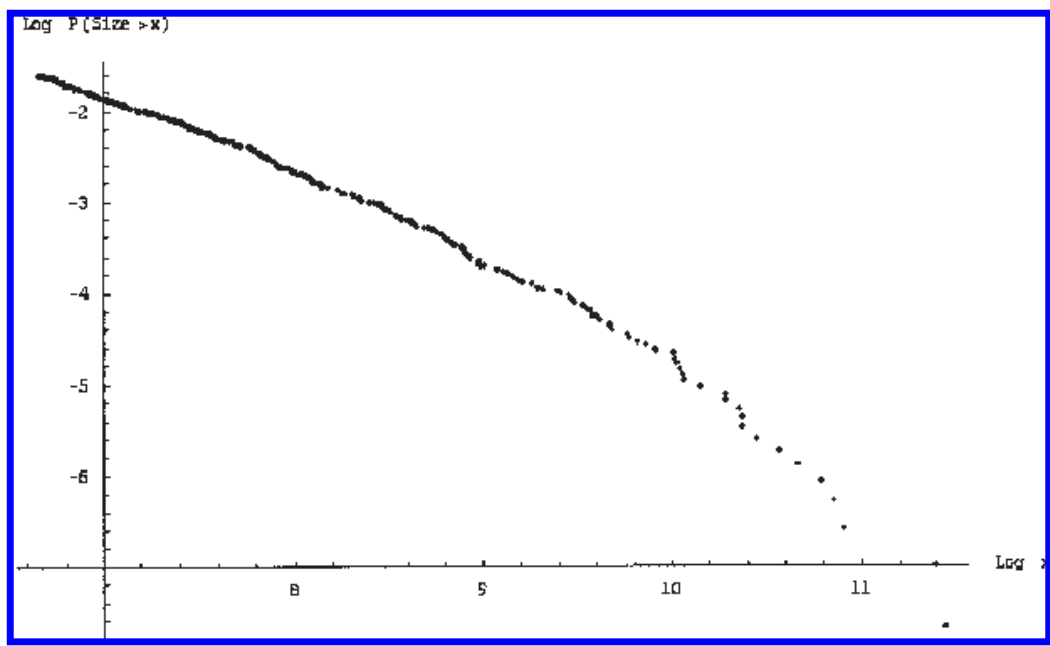

FIGURE VII

Cumulative distribution of the size (assets under management) of the top mutual funds in 1999. Source: Center for Research on Security Prices.

set of large market participants. In the meantime, we consider evidence such as Figure VI as supportive of a linear relationship between volume and squared return. However, it is possible that the true relationship is different, or may vary from market to market. This is why we present a theory with a general curvature $\gamma$.

II.D. The Power Law Distribution of the Size of Large Investors: $\zeta_{S} \simeq 1$

It is highly probable that substantial trades are generated by very large investors. This motivates us to investigate the size distribution of market participants. A power-law formulation,

$$
P(S>x) \sim 1 / x^{\zeta S},
$$

often yields a good fit.

The exponent $\zeta_{S} \simeq 1$, often called Zipf's law, is particularly common. This relation is true for both cities [Zipf 1949; Gabaix and Ioannides 2004] and firms [Axtell 2001; Okuyama, Takayasu, and Takayasu 1999; Fujiwara, Di Guilmi, Aoyama, Gallegati, and Souma 2004]. If the distribution of firms in general follows Zipf's law, it is plausible to hypothesize that the distribution of money management firms in particular follows Zipf's law. Indeed, Push- 
kin and Aref [2004] find that this is the case for U. S. bank sizes, measured by assets under management.

We investigate firms for which money management is the core business: mutual funds. ${ }^{28}$ We use CRSP to obtain the size (dollar value of assets under management) of all mutual funds ${ }^{29}$ from 1961-1999 (see Figure VII). For each year $t$, we estimate the power-law exponent $\zeta$ of the tail distribution (20 percent cutoff) via OLS. We find an average coefficient $\overline{\zeta_{t}}=1.10$, with a standard deviation across years of $0.08 .^{30}$ The Hill estimator technique gives a mean estimate $\overline{\zeta_{t}}=0.93$ and a standard deviation of 0.07 . Hence we conclude that, to a good approximation, mutual fund sizes follow a power-law distribution with exponent

$$
\zeta_{S} \simeq 1 \text {. }
$$

For this paper we can take this distribution of the sizes of mutual funds as a given. It is, in fact, not difficult to explain. One can apply the explanations given for cities [Simon 1955; Gabaix 1999; Gabaix and Ioannides 2004] to mutual funds. Suppose that the relative size $S_{i t}$ of a mutual fund $i$ follows a random growth process $S_{i t}=S_{i, t-1}\left(1+\varepsilon_{i t}\right)$, with $\varepsilon_{i t}$ i.i.d. and mean 0. Add a minor element of friction to small funds to ensure a steady state distribution; for instance, very small funds are terminated and are replaced by new funds. Then, this steady state distribution follows Zipf's law with $\zeta_{S}=1 .^{31}$

Gabaix, Ramalho, and Reuter [2005] develop this idea and show that these assumptions are verified empirically. This means that the random growth of mutual funds generically leads their size distribution to satisfy Zipf's law, $\zeta_{S}=1$.

It is only in the past 30 years that mutual funds have come to represent a large part of the marketplace. It would be interesting to have evidence on the size distribution of financial institutions before mutual funds became important. For instance, pension

28. Here we sketch the main findings. Gabaix, Ramalho, and Reuter [2005] present much more detail.

29 . The $x$ funds of Fidelity, for instance, count as $x$ different funds, not as one big "Fidelity" fund.

30 . We cannot conclude that the standard deviation on our mean estimate is $0.08(1999-1961+1)^{-.5}$. The estimates are not independent across years, because of the persistence in mutual fund sizes.

31. It may be useful to give a short proof. Suppose that the process is $d S_{t}=$ $S_{t} \sigma d B_{t}$. The steady state density $p(S)$ satisfies the forward Kolmogorov equation $0=\partial_{t} p=(1 / 2)\left(d^{2} / d S^{2}\right)\left(\sigma^{2} S^{2} p(S)\right)$. This implies that $p(S)=k / S^{2}$ for a constant $k$, and a cumulative distribution $P(S>x)=k / x$, i.e., Zipf's law. 
funds of corporations are likely to follow Zipf's law, as the number of employees in firms follow Zipf's law.

The evidence we present here is necessarily tentative. Estimating a power law with a relatively small number of points is very difficult, and all estimators require somewhat arbitrary parameters [Embrechts, Kluppelberg, and Mikosch 1997]. Furthermore, we had access to only a subset of the participants in the U. S. market. Other important participants are hedge funds, pension funds, and proprietary trading desks, and foreign institutions. It would be useful to weight the funds by their leverage and their annual turnover. Nevertheless, given that Zipf's law (equation (9)) has been found to describe the size of many other entities, such as banks and firms in general, and appears to describe well the upper tail of the empirical distribution of mutual funds, we view equation (9) as a good benchmark.

\section{II.E. Summary and Paradoxes}

The facts summarized in this subsection present important challenges. First, economic theories have difficulties in explaining the power-law distribution of returns, as the efficient market theory, and GARCH models, need to be fine-tuned to explain why the distribution of returns would have an exponent of 3 .

Second, it is surprising that the Pareto exponent of trading volume is $\zeta_{q} \simeq 1.5$, while that of institution size is $\zeta_{S} \simeq 1$. In models with frictionless trading, all agents have identical portfolios and trading policies, except that they are scaled by the size $S$ of the agents (which corresponds to wealth). Hence frictionless trading predicts that the distribution of trading volume of a given stock should reflect the distribution of the size of its investors; i.e., $\zeta_{q}=\zeta_{S} \simeq 1 .{ }^{32}$ However, we find that $\zeta_{q}>\zeta_{S} \cdot{ }^{33}$ A likely cause is the cost of trading; large institutions trade more prudently than small institutions, because price impact is monotonically increasing in trade size.

Finally, the basic price impact model [Kyle 1985] predicts a

32. Solomon and Richmond [2001] have proposed a model that relies on a scaling exponent of wealth $\zeta_{S}=3 / 2$. We are sympathetic to this approach that links wealth to volumes. In the present study we use the size distribution of institutions, rather than individual wealth, because most very large trades are likely to be done by institutions rather than by private individuals. Also, the Pareto exponent of wealth and income is quite variable (e.g., Davies and Shorrocks [2000] and Piketty and Saez [2003]).

33. The lower the power-law exponent, the fatter the tails of the variable. See Appendix 1. 
linear relation between returns and volume, which would imply $\zeta_{r}=\zeta_{q}$. To explain why $\zeta_{q} / \zeta_{r}$ is close to $1 / 2$, we require a model with curvature of price impact $\gamma=1 / 2$.

We now present a model that attempts to resolve the above paradoxes. ${ }^{34}$

\section{The Model}

We consider a large fund in a relatively illiquid market. We first describe a rudimentary model for the price impact of its trades. Next, we link the various power law exponents; this represents the core contribution of this paper. One could employ different microfoundations for price impact without changing our conclusions.

\section{III.A. A Simple Model to Generate a Power Law Price Impact}

Before presenting, in subsection III.B, the core of the model, we first present a simple microfoundation for the square root price impact. The basic model of Kyle [1985] predicts a linear price impact. Subsequent models, such as Seppi [1990], Barclay and Warner [1993], and Keim and Madhavan [1996], generate a concave impact in general. Zhang [1999] and Gabaix, Gopikrishnan, Plerou, and Stanley [2003] produce a square root function in particular. ${ }^{35}$ The model used in this section is a formalized version of a useful heuristic argument, sometimes called the "Barra model" of Torre and Ferrari [Barra 1997].

We consider a single risky security in fixed supply, with a price $p(t)$ at time $t$. The large fund ("he") buys or sells the security from a liquidity supplier ("she"). The timing of the model is as follows:

At time $t=0$, the fund receives a signal $M$ about mispricing: $M<0$ is a sell signal; $M>0$ is a buy signal. Without loss of generality, we study a buy signal. The analysis is symmetric for a sell signal.

At $t=1-2 \varepsilon$ ( $\varepsilon$ is a small positive number), the fund

34. Gabaix, Gopikrishnan, Plerou, and Stanley [2003] present a reduced form of some elements of the present article.

35. Gabaix, Gopikrishnan, Plerou, and Stanley [2003] predicts that a trade of size $V$ will be traded into $N=V^{1 / 2}$ smaller chunks. This has the advantage of generating a power-law distribution of the number of trades with exponent $\zeta_{N}=$ $2 \zeta_{V}=3$, which is close to the empirical value [Plerou, Gopikrishnan, Amaral, Gabaix, and Stanley 2000]. We did not keep it in the current model, because we wanted to streamline the microfoundation of price impact. 
negotiates a price with the liquidity supplier. For simplicity, we assume liquidity provision is competitive so that the fund has full bargaining power. The liquidity supplier sells to the fund the quantity $V$ of shares, at a price $p+R$, where $p=p(1-2 \varepsilon)$ is the price before impact and $R$ is the price concession, or full price impact. ${ }^{36}$

At $t=1-\varepsilon$, the transaction is announced to the public.

At $t=1$, the price jumps to $p(1)=p+\pi(V)$, where $\pi(V)$ is the permanent price impact. The difference between $\pi$ and $R$ is the temporary price impact $\tau=R-\pi$. Equilibrium will determine the value of the permanent price impact $\pi(V)$ and the price concession $R(V)$.

From $t=1$ onwards, the price follows a random walk with volatility $\sigma$ :

$$
p(t)=p+\pi(V)+\sigma B(t),
$$

where $B$ is a standard Brownian motion with $B(1)=0$. Also, at $t=1$, the liquidity supplier starts replenishing her inventory. She continuously meets sellers who are willing to sell her a quantity $\bar{V} d t$ of the stock at price $p(t)$ : she is a price taker as she can credibly assure that she is not informed. The liquidity supplier continues to buy shares until her inventory is fully replenished, which happens after a time $T=V / \bar{V}$. The price continues to evolve according to (10). ${ }^{37}$

The liquidity provider benefits from the temporary price impact $\tau$, but then faces price uncertainty as she replenishes her inventory [Grossman and Miller 1988]. To evaluate these effects, we assume that the liquidity provider has the following mean variance utility function on the total amount $W$ of money earned during the trade:

$$
U=E[W]-\lambda[\operatorname{var}(W)]^{\delta / 2},
$$

36. To keep the mathematics simple, the impact is additive, and the price otherwise follows a random walk. It is easy, though cumbersome, to make the price impact proportional and the log price follow a random walk. Our conclusions about the power-law exponents would not change.

37. We wish to add two comments about the timing of the model. In our model, the large fund trades in one block (at time $t=1$ ), and the liquidity provider trades in many smaller chunks (at time $t \in(1,1+T]$ ). Many alternative timing assumptions would leave the scaling relations unchanged (12), with the same $\gamma$. Also, the exchange between the large fund and the liquidity supplier is an "upstairs" block trade. In an upstairs trade, the initiator typically commits not to repeat the trade too soon in the future. This prevents many market manipulation strategies that might otherwise be possible with a nonlinear price impact, such as those analyzed by Huberman and Stanlz [2004]. 
with $\lambda>0$ and $\delta>0$. The liquidity supplier requires compensation equal to $\lambda \sigma^{\delta}$ to bear a risk of standard deviation $\sigma$, i.e., has "סth order risk aversion." 38 With standard mean-variance preferences, $\delta=2$. In many cases, a better description of behavior is first-order risk aversion, which corresponds to $\delta=1$. $^{39}$

One justification for first-order risk aversion comes from psychology. Prospect theory [Kahneman and Tversky 1979] presents psychological evidence for this behavior, which has also been formalized in disappointment aversion [Gul 1991; Backus, Routledge, and Zin 2005]. Second, first-order risk aversion is frequently needed to calibrate quantitative models, such as Epstein and Zin [1990] and Barberis, Huang, and Santos [2001]. A third justification is institutional, as (11) can reflect a value at risk penalty, where $\lambda$ is the size of the penalty, and $\operatorname{var}(W)^{1 / 2}$ is proportional to the value at risk. Another institutional justification is via the Sharpe ratio. If a trader uses a rule to accept trades if and only if their Sharpe ratio is greater than $\lambda$, then he will behave as if he exhibits first-order risk aversion.

Proposition 1 . The setup of this section generates the temporary price impact function:

$$
\tau(V)=H V^{\gamma}
$$

with $H=\lambda \sigma^{\delta} /(3 \bar{V})^{\delta / 2}$ and

$$
\gamma=3 \delta / 2-1 .
$$

For future reference, it is useful to state separately our central case.

PROPOSITION 2. If the liquidity provider is first-order risk averse, then the price impact increases with the square root of traded volume:

$$
\gamma=1 / 2
$$

and

$$
\tau(V)=\lambda \sigma\left(\frac{V}{3 \bar{V}}\right)^{1 / 2}
$$

38. Essentially all nonexpected utility theories need a postulate on how different gambles are integrated. Here, we assume that the liquidity provider evaluates individually the amount $W$ earned in the trade.

39. The model also generates a square root price impact with a different specification that generates first-order risk aversion, for instance the loss averse utility function: $U=E[\max (W, 0)]+\Lambda E[\min (W, 0)]$ with $\Lambda>1$. 
$\sigma$ is the daily volatility of the stock, and $\lambda$ the risk aversion of the liquidity provider.

In practice, $\bar{V}$ is likely to be proportional to the daily trading volume. Hence the scaling predictions of equation (13) can be almost directly examined.

The proof is in Appendix 2. The intuition is that the liquidity provider needs a time $T=V / \bar{V}$ to buy back the $V$ shares. During that time, the price diffuses at a rate $\sigma$. Hence the liquidity provider faces a price uncertainty with standard deviation $\sigma \sqrt{T} \sim \sigma \sqrt{V}$. If the liquidity provider is first-order risk averse, the price concession $\tau$ is proportional to the standard deviation, hence $\tau \sim \sigma \sqrt{V}$, i.e., equation (13).

To close the model, we need to determine both the permanent and the full price impact. The determination of these two variables typically depends on the fine details of the information structure processed by the other market participants. We use a somewhat indirect route, which drastically simplifies the analysis.

Assumption 1. We assume that the market uses a linear rule to determine the full price impact,

$$
R(V)=B \tau(V)
$$

for some $B>0$. Subsection IV.A presents conditions under which the linear rule (14) is actually optimal.

Assumption 1 closes the price impact part of the model.

Proposition 3. The above setup generates the price concession function

$$
R(V)=h V^{\gamma},
$$

where $h=B H$, and $H$ and $\gamma$ are determined in Propositions 1 and 2 .

\section{III.B. The Core Model: Behavior of a Large Fund}

We now lay out the core of our model. The fund periodically receives signals about trading opportunities, which indicate that the excess risk-adjusted return on the asset is $s_{t} M_{t} \tilde{C} . s_{t}, M_{t}$ and $\tilde{C}$ are independent. $s_{t}= \pm 1$ is the sign of the mispricing. $M_{t}$ is the expected absolute value of the mispricing. $M_{t}$ is drawn from a distribution $f(M)$, which we assume to be not too fat-tailed. 
Assumption 2. We assume that $M$ is not too fat-tailed: $E\left[M^{1+1 / \gamma}\right]$ $<\infty$.

The model misspecification risk $\tilde{C}$ captures uncertainty over whether the perceived mispricing is in fact real. For example, the fund's predictive regressions may result from data mining, or the mispricing may have since been arbitraged away. $\tilde{C}$ can take two values, 0 and $C^{*}$. If $\tilde{C}=0$, the signals the fund perceives are pure noise, and the true average return on the perceived mispricings is 0 . If $\tilde{C}=C^{*}$, the mispricings are real. We specify $E[\tilde{C}]=1$, so that $M$ represents the expected value of the mispricing.

The fund has $S$ dollars in assets. If it buys a volume $V_{t}$ of the asset, and pays a price concession $R\left(V_{t}\right)$, the total return of its portfolio is

$$
r_{t}=V_{t}\left(\tilde{C} M_{t}-R\left(V_{t}\right)+u_{t}\right) / S,
$$

where $u_{t}$ is mean zero noise.

If the model is wrong, expected returns are

$$
E\left[r_{t} \mid \tilde{C}=0\right]=-V_{t} R\left(V_{t}\right) / S .
$$

We assume that the manager has a concern for robustness. He does not want his expected return to be below some value $-\Lambda$ percent if his trading model is wrong. Formally, this means that

$$
E\left[r_{t} \mid \tilde{C}=0\right] \geq-\Lambda .
$$

Equation (18) can be justified in several ways. One is a psychological attitude toward model uncertainty, developed in depth by Gilboa and Schmeidler [1989] and Hansen and Sargent [2005]. Second, equation (18) is a useful rule of thumb, which can be applied without requiring detailed information about the fine details of model uncertainty. A third explanation is delegated management [Shleifer and Vishny 1997]. If trader ability is uncertain, investors may wish to impose a constraint such as equation (18) to prevent excessive trading.

To simplify the algebra, we assume that, subject to the robustness constraint, the manager wants to maximize the expected value of his excess returns $E[r] .{ }^{40}$ We now summarize this.

40. One might prefer the formulation $\max _{V(M, S)} E[u(r)]$ subject to $E[u(r) \mid C=0] \geq u(-R)$, with a concave utility $u$. Fortunately, this does not change the conclusions in many instances, such as $u(r)=-e^{-\alpha r}, \alpha>0$. On the 
Definition 1. Suppose that the fund has $S$ dollars under management. The fund's optimal policy is a function $V(M, S)$ that specifies the quantity of shares $V$ traded when the fund perceives a mispricing of size $M$. It maximizes the expected returns $E\left[r_{t}\right]$ subject to the robustness constraint (18):

$$
\max _{V(M, S)} E\left[r_{t}\right] \text { subject to } E\left[r_{t} \mid \tilde{C}=0\right] \geq-\Lambda \text {. }
$$

\section{III.C. Optimal Strategy and Resulting Power Law Exponents}

We can now derive the large fund's strategy. Given equation (16), Definition 1 is equivalent to

$$
\max _{V(M, S)} \frac{1}{S} \int_{0}^{\infty} V(M, S)(M-R(V(M, S))) f(M) d M
$$

subject to

$$
\frac{-1}{S} \int_{0}^{\infty} V(M, S) R(V(M, S)) f(M) d M \geq-\Lambda .
$$

Appendix 2 establishes the following proposition.

Proposition 4. If constraint (18) binds, the optimal policy for a fund of size $S$ perceiving a mispricing of size $M$ is to trade a volume

$$
V(M, S)=v M^{1 / \gamma} S^{1 /(1+\gamma)} .
$$

The price change after the trade is

$$
R(M, S)=h v^{\gamma} M S^{\gamma /(1+\gamma)}
$$

for a positive constant $v$, defined in equation (48), which is increasing in $\Lambda$ and decreasing in $h$.

Equation (21) means that price movements reflect both the intensity of the perceived mispricing $M$, and the size of the fund $S$. Concretely, a large price movement can come from an extreme signal or the trade of a large fund [Easley and O'Hara 1987].

In the remaining analysis, we assume that (18) holds over the support of $S$.

other hand, with a nonlinear function $u$ the derivations are more complex, as they rely on asymptotic equalities, rather than exact equalities. To keep things simple, we use the linear representation (19). 
Assumption 3. The robustness constraint (18) binds for all funds in the market above a certain size.

A simple calibration presented in Appendix 2 shows that Assumption 3 holds for funds that manage less than $S^{*}=\$ 21$ trillion dollars. Assumption 3 is not very stringent. Alternatively, subsection IV.C shows a way to ensure Assumption 3 without any finite size effects. ${ }^{41}$

We next derive the distribution of volume and price changes.

Proposition 5. The traded volume and the price changes follow power-law distributions with respective exponents:

$$
\begin{aligned}
& \zeta_{V}=\min \left[(1+\gamma) \zeta_{S}, \gamma \zeta_{M}\right] \\
& \zeta_{R}=\min \left[\left(1+\frac{1}{\gamma}\right) \zeta_{S}, \zeta_{M}\right] .
\end{aligned}
$$

Equation (22) implies that the distribution of price movements reflects both the "news" (perhaps coming from proprietary analysis), as reflected in $M$, as well as the size $S$ of the agents that act on the news. Equation (23) illustrates the resulting exponent. In equilibrium, it is the fatter of the two tails of signals and sizes that matters. Mathematically, this comes from the properties (38) of power laws: the tail exponent of the product of two independent random variables $X_{1}$ and $X_{2}$ is equal to the tail exponent of the more fat-tailed variable, i.e., is the lower of the exponents of $X_{1}$ and $X_{2}$. Economically, this means that the polar case, where large investors affect the tail of trading volume, is captured when $\zeta_{M}>$ $(1+1 / \gamma) \zeta_{S}$. Then, we get

$$
\begin{gathered}
\zeta_{V}=(1+\gamma) \zeta_{S} \\
\zeta_{R}=\left(1+\frac{1}{\gamma}\right) \zeta_{S} .
\end{gathered}
$$

Equation (23) then means that, when there is a very large movement, it is more likely to come from the actions of a very large institution (the $S$ term), rather than an objectively important piece of news (the $M$ term). This potential importance of a large

41. Such cutoffs are generally present when handling power laws, and are sometimes called "border" or "finite size" effects. The cutoff affects only very little predictions. For instance, it affects the power-law exponent of returns only by a factor $10^{-3}$ if a large fund has a size $S=10^{-3} S^{*}$, which is a plausible empirical order of magnitude. 
institution may explain why, during the Long Term Capital Management crisis, the October 1987 crash, and the events studied by Cutler, Poterba, and Summers [1989], prices moved in the absence of significant news items. In the context of our theory, the extreme returns occurred because some large institutions wished to make substantial trades in a short time period.

Proposition 5 says that when the distribution of the size of institutions is more fat-tailed, volume and returns are also more fat-tailed. However, when the curvature $\gamma$ of price impact is smaller, returns are less fat-tailed, but volumes are more fattailed. The reason is that large institutions trade more moderately when the price impact is steeper. We now apply Proposition 5 to our baseline values.

PRoposition 6. With a square root price impact $(\gamma=1 / 2)$ and Zipf's law for financial institutions $\left(\zeta_{S}=1\right)$, volumes and returns follow power-law distributions, with respective exponents of $3 / 2$ and 3 :

$$
\begin{aligned}
& \zeta_{V}=3 / 2 \\
& \zeta_{R}=3 .
\end{aligned}
$$

These exponents are the empirical values of the distribution of volume and returns.

Proposition 6 captures our explanation of the origins of the cubic law of returns, and the half-cubic law of volumes. Random growth of mutual funds leads to Zipf's law of financial institutions, $\zeta_{S}=1$. The model in subsection III.A leads to a power law price impact with curvature $\gamma=1 / 2$. As large funds wish to lessen their price impacts, their trading volumes are less than proportional to their size. This generates a power-law distribution of the size of trades that is less fat-tailed than the size distribution of mutual funds. The resulting exponent is $\zeta_{V}=3 / 2$, which is the empirical value. Trades of large funds create large returns, and indeed the power law distribution of returns with exponent $\zeta_{r}=3$.

\section{RoBustness AND Extensions}

\section{IV.A. Permanent versus Transitory Price Impact}

So far we have analyzed the full price impact $R$, which is the sum of a permanent component $\pi$ and transitory component $\tau$ : 
$R=\pi+\tau$. We provide a sufficient condition that will ensure that the permanent and the full price impacts are proportional. In a Bayesian framework, the price impact must come from an inference, which from Proposition 4 is

$$
\pi(V)=E\left[M \mid h V^{\gamma}=h v^{\gamma} M^{\gamma} S^{\gamma /(1+\gamma)}\right] .
$$

The conditional expectation (28) is complicated and can be nonlinear. It is difficult to see how agents would apply Bayes' rule to compute (28), which requires knowing the distribution of $M$, and $M$ is not a directly observable quantity. However, these difficulties vanish in a class of cases-when agents use (28) with the belief that $\zeta_{M} \leq \zeta_{R}$. The case where they believe $\zeta_{M}=\zeta_{R}$ is particularly plausible. If one does not know the distribution of mispricings perceived by other agents, one might hypothesize that it is close to the distribution of returns. This motivates the following proposition.

Proposition 7. Suppose that updaters performing (28) believe $\zeta_{M} \leq \zeta_{R}$. Then, the exponent $\zeta_{\pi}$ of the permanent price impact is equal to the exponent $\zeta_{R}$ of the full price impact, and is given by Proposition 5:

$$
\zeta_{\pi}=\zeta_{R}=\min \left[\left(1+\frac{1}{\gamma}\right) \zeta_{S}, \zeta_{M}\right] .
$$

If the updaters believe $\zeta_{M}<\zeta_{R}$, there is a constant $b>0$ such that, for large volumes $V$, the permanent price impact is

$$
\pi(V)=E[M \mid V] \sim b V^{\gamma} .
$$

If updaters performing (28) believe $\zeta_{M}=\zeta_{R}$, then $\pi(V)=$ $V^{\gamma} L(V)$, where $L$ is a "slowly varying" function that varies more slowly than any polynomial (see Appendix 1).

Proposition 7 presents sufficient conditions for $\pi(V)$ to preserve the power-law price impact under Bayesian updating, and thus to justify Assumption 1.

\section{IV.B. Multiple Stocks}

The model can easily be extended to multiple stocks. Suppose that stock $i$ has a power-law impact $R_{i}(V)=h_{i} V^{\gamma}$, that the signal $M s$ are independent across stocks, and the model misspecification 
risk $\tilde{C}$ is common across stocks. ${ }^{42}$ The trader's program is to maximize the expected profit from trading over all stocks:

$$
\max _{V_{i}\left(M_{i}, S\right), i=1, \ldots, n} \frac{1}{S} \sum_{i} \int_{0}^{\infty} V_{i}\left(M_{i}, S\right)\left(M_{i}-R_{i}\left(V_{i}(M, S)\right)\right) f_{i}\left(M_{i}\right) d M_{i},
$$

subject to the robustness constraint that he does not lose more than $\Lambda$ percent in price impact costs:

$$
\frac{1}{S} \sum_{i} \int_{0}^{\infty} V_{i}\left(M_{i}\right) R_{i}(V(M)) f_{i}\left(M_{i}\right) d M_{i} \leq \Lambda .
$$

Following the proof of the main proposition, one can show that the solution is $h_{i} V_{i}\left(M_{i}, S\right)^{\gamma}=K M_{i} S^{\gamma /(1+\gamma)}$, where $K$ does not depend on $i$ and $S$. Hence, the power law exponents derived in Propositions 5-6 follow.

\section{IV.C. Different Quality of Signals across Firms}

We now allow the quality of signal $M$ to differ across funds, and show that this does not affect our results. We assume that fund $f$ receives signals distributed according to $M=\chi_{f} m$, where $\chi_{f}$ is the quality of the fund's signals, and the distribution of $m$ is the same across funds. Following the proof of Proposition 4, the optimal trading quantity of a fund of size $S$ is still, for a constant $K=(\Lambda / h)^{1 /(1+\gamma)}$ :

$$
V(m, S)=K \frac{M^{1 / \gamma} S^{1 /(1+\gamma)}}{E\left[M^{(1+\gamma) / \gamma}\right]^{1 /(1+\gamma)}}=K \frac{m^{1 / \gamma} S^{1 /(1+\gamma)}}{E\left[m^{(1+\gamma) / \gamma}\right]^{1 /(1+\gamma)}},
$$

as $M=\chi_{f} m$. The average quality $\chi_{f}$ of the signals disappears. Hence, one still obtains $\zeta_{V}=\min \left[(1+\gamma) \zeta_{S}, \gamma \zeta_{m}\right]$ and $\zeta_{R}=\zeta_{V} / \gamma$.

In general, one expects larger firms to have a higher $\chi$. For instance, if signals are generated according to a production function $\chi(F)=F^{\mathrm{\kappa}}$, where $F$ denotes investment in research, then the optimal investment for a fund satisfies $\max _{F} C F^{\mathrm{\kappa}} S^{\gamma /(1+\gamma)}-F$, for a constant $C$. Hence $F \sim S^{\gamma /[(1-\kappa)(1+\gamma)]}$ and the quality of signals is $\chi \sim S^{\theta}$ for $\theta=\gamma \kappa /[(1-\kappa)(1+\gamma)]$.

This framework allows us to provide a microfoundation for Assumption 3 without any upper cutoff. The proof of Proposition 4 shows that Assumption 3 holds if

42. It is easy to verify that $\tilde{C}$ could also be specific to each stock, or to each one of different classes of stocks. 


$$
\begin{aligned}
S & <\frac{E\left[M^{1+1 / \gamma}\right][(1+\gamma)]^{-(1+1 / \gamma)}}{h^{1 / \gamma} \Lambda} \\
& =\frac{E\left[m^{1+1 / \gamma}\right][(1+\gamma)]^{-(1+1 / \gamma)}}{h^{1 / \gamma} \Lambda} S^{\theta[(1+\gamma) / \gamma]} \sim S^{\kappa /(1-\kappa),}
\end{aligned}
$$

which holds if к $>1 / 2$ and $S$ is large enough. Thus, Assumption 3 is automatically verified if the production function of market research rises faster than $\chi=F^{1 / 2}$.

\section{IV.D. Discussion and Questions for Future Research}

Is it reasonable to believe that there are institutions large enough to cause the power-law distribution of returns? In view of the empirical facts, we believe so. The large volumes in Figure V, which can be 1000 times bigger than the median trades, must come from very large traders. They are also associated with extreme price movements (Figure VI). However, a natural analysis would be to investigate directly whether extreme movements without news [Cutler, Poterba, and Summers 1989] are caused by a small number of large institutional investors. The growing availability of databases that track individual trades may allow such a study to be conducted in the near future. Note that the existence of prime movers does not preclude that, subsequently, many traders will move in the same way. Quantifying the importance of idiosyncratic movements of large trades versus correlated movements of beliefs of most traders would be interesting. ${ }^{43}$

One prominent example of a large fund disrupting the market is Long Term Capital Management. Its collapse created a volatility spike that did not subside for several months. Our contribution is a model of the initial impulse-the form and the power-law distribution of the initial disruption by a large trade. We leave to future research the important task of modeling the specifics of the cascade that followed the initial impulse. ${ }^{44} \mathrm{We}$ speculate that the empirical facts we present, and our baseline model of initial impulses, will be useful for this future research.

A second example is the Brady [1988] report on the 1987

43. Gabaix [2005] finds that the idiosyncratic movements of large firms explain a substantial fraction of macroeconomic activity, and Canals, Gabaix, Vilarrubia, and Weinstein [2005] find that idiosyncratic shocks explain a large fraction of international trade.

44. Abreu and Brunnermeier [2003], Bernardo and Welch [2004], Gennotte and Leland [1900], Romer [1993], and Greenwald and Stein [1991] also present elements for a theory of crashes. 
crash. On the crash day of Monday, October 19, 1987, "this trading activity was concentrated in the hands of surprisingly few institutions.... Sell programs by three portfolio insurers accounted for just under $\$ 2$ billion in the stock market. ... Block sales by a few mutual funds accounted for about $\$ 900$ million of stock sales," on a total of $\$ 21$ billion traded [p. v] and "One portfolio insurer alone sold $\$ 1.3$ billion" [p. III-22]. In the first half hour of trading, "roughly 25 percent of the volume . . . came from one mutual fund group" [p. 30]. The report concludes that "much of the selling pressure was concentrated in the hands of surprisingly few institutions. A handful of large investors provided the impetus for the sharpness of the decline" [p. 41]. Of course, some of the investors in the Brady report are program traders, which amplify existing movements, rather than cause them. Also, our model is still too limited to allow the rich dynamic analysis suggested by the Brady report. Nonetheless, the evidence from the report is strongly suggestive of the hypothesis that a few traders move a relatively illiquid market.

Our theory suggests a number of research angles. First, it would be desirable to study fully dynamic extensions of the model. The analysis becomes much more difficult (see e.g., Vayanos [2001] and Gabaix, Gopikrishnan, Plerou, and Stanley [2003]), but the simplicity of the empirical distributions suggests that a simple dynamic theory of large events may be within reach. ${ }^{45}$

Second, it would be interesting to study the distribution of fund "effective" size (assets multiplied by leverage) across classes of stocks. Proposition 5 predicts that the more fat-tailed the size distribution of traders, the more fat-tailed the distributions of volume and returns. Investigating this prediction directly might explain a cross-sectional dispersion of power-law exponents.

Third, our model predicts that the total price impact cost paid by a fund of size $S$ will be proportional to $S$, and that the total volume traded with sizable price impact will be proportional to $S^{1 /(1+\gamma)}$. Testing this proposition directly would be useful.

Fourth, the model suggests a particularly useful functional pirical investigations of the dynamic relations between trading and returns. 
form for "illiquidity," which corresponds more closely to the prefactor of Proposition $3^{46}$ :

$$
\begin{gathered}
\hat{H}=E\left[\frac{\left|r_{t}\right|}{V_{t}^{\gamma}}\right] \\
\hat{H}^{\prime}=\frac{\operatorname{cov}\left(\left|r_{t}\right|, V_{t}^{\gamma}\right)}{\operatorname{var} V_{t}^{\gamma}} .
\end{gathered}
$$

Again, the evidence, and some models, suggests $\gamma=1 / 2$, but other values may prove better suited. Expressions (31) and (32) are likely to be more stable than other measures. Indeed, volume is a fat-tailed variable (it has infinite variance), so using a square root of volume is likely to yield a more stable measure than volume itself. Furthermore, the model also suggests that $\hat{H}$ and $\hat{H}^{\prime}$ will be proportional to $\sigma / M^{1 / 2}$, where $\sigma$ is the volatility of the stock and $M$ is its market capitalization.

Fifth, our approach suggests a way to estimate the power-law exponent of price impact, $\gamma$, and the power-law exponent of the distribution of financial institutions, $\zeta_{S}$, for instance across markets. One first estimates separately the power-law exponents of volumes and returns, $\zeta_{q}$ and $\zeta_{r}$. Then one defines the estimators $\hat{\gamma}$ and $\hat{\zeta}_{S}$ by

$$
\begin{gathered}
\hat{\gamma}=\frac{\zeta_{q}}{\zeta_{r}} \\
\frac{1}{\hat{\zeta}_{S}}=\frac{1}{\zeta_{r}}+\frac{1}{\zeta_{q}} .
\end{gathered}
$$

Proposition 5 indicates that these are consistent estimates of $\gamma$ and $\zeta_{S}$ in the polar case where $\zeta_{M}>\zeta_{S}(1+\gamma) / \gamma \cdot{ }^{47}$

Finally, the theory makes predictions about the comovements in returns, volume, and signed volume (the sum of volumes traded on a price increase minus volume traded on a price decrease). Its variant in Gabaix, Gopikrishnan, Plerou, and Stanley [2003] adds predictions in the number of trades and signed number of trades. The results show nonlinear patterns, and the results, reported in Figure 3 of Gabaix, Gopikrishnan, Plerou, and

46. One could even calculate the two expressions only for volumes above a certain threshold, e.g., the mean volume.

47. It is tempting to call equation (34) a "reciprocity law" that holds irrespective of $\gamma$. 
Stanley [2003], show a quite encouraging fit between theory and data.

\section{CONCLUSION}

This paper proposes a theory in which large investors generate significant spikes in returns and volume. We posit that the specific structure of large movements is due to the desire to trade of sizable institutional investors, stimulated by news. The distribution of fund sizes, coupled with large traders' moderation of their trading volumes and a concave price impact function, generates the Pareto exponents 3 and $3 / 2$ for the distribution of returns and volumes.

We introduce some new questions that finance theories should answer. Matching, as we do, the quantitative empirical regularities outlined here (in particular explaining the exponents of approximately 3 and $3 / 2$ from first principles rather than by assumption) should be a sine qua non criterion for the admissibility of a model of volume and volatility. We hope that the regularities we established will constrain and guide future theories. Given its simple structure, the present model might be a useful point of departure for thinking about these issues.

Appendix 1: Some Power Law Mathematics

\section{A. Definitions}

We present here some basic facts about power-law mathematics, and show how their aggregation properties make them especially interesting for both theoretical and empirical work. They also show how our predictions are robust to other sources of noise.

A random variable $X$ has power-law behavior if there is a $\zeta_{X}>0$ such that the probability density $p(x)$ follows:

$$
p(x) \sim \zeta_{X} C / x^{\zeta X+1}
$$

for $x \rightarrow \infty$, and a constant $C$. This implies (e.g., Resnick [1987, p. 17]) that the "counter-cumulative" distribution function follows:

$$
P(X>x) \sim C / x^{\zeta x} .
$$


A more general definition is that there is a "slowly varying"48 function $L(x)$ and a $\zeta_{X}$ such that $p(x) \sim L(x) / x^{\zeta_{X}+1}$, so that the tail follows a power law up to slowly varying corrections.

$\zeta_{X}$ is the (cumulative) power-law exponent of $X$. A lower exponent means fatter tails: $\zeta_{X}<\zeta_{Y}$ implies that $X$ has fatter tails than $Y$, hence the large $X$ 's are (infinitely, at the limit) more frequent than large $Y$ 's.

If $\alpha$ is a constant, $E\left[|X|^{\alpha}\right]=\infty$ for $\alpha>\zeta_{X}$, and $E\left[|X|^{\alpha}\right]<\infty$ for $0 \leq \alpha<\zeta_{X}$. For instance, if returns have power-law exponents $\zeta_{r}=3$, their kurtosis is infinite, and their skewness borderline infinite. ${ }^{49}$ If all moments are finite (e.g., for a Gaussian distribution), the formal power-law exponent is $\zeta_{X}=\infty$.

\section{B. Transformation Rules}

Power laws have excellent aggregation properties. The property of being distributed according to a power law is conserved under addition, multiplication, polynomial transformation, min, and $\max$. The general rule is that, when we combine two powerlaw variables, "the fattest (i.e., the one with the smallest exponent) power law dominates." Indeed, for $X_{1}, \ldots, X_{n}$ independent random variables, and $\alpha$ a positive constant, we have the following formulas:

$$
\begin{gathered}
\zeta_{X_{1}+\ldots+X_{n}}=\min \left(\zeta_{X_{1}}, \ldots, \zeta_{X_{n}}\right) \\
\zeta_{X_{1} \ldots X_{n}}=\min \left(\zeta_{X_{1}}, \ldots, \zeta_{X_{n}}\right) \\
\zeta_{\max \left(X_{1}, \ldots, X_{n}\right)}=\min \left(\zeta_{X_{1}}, \ldots, \zeta_{X_{n}}\right) \\
\zeta_{\min \left(X_{1}, \ldots, X_{n}\right)}=\zeta_{X_{1}}+\ldots+\zeta_{X_{n}} \\
\zeta_{\alpha X}=\zeta_{X} \\
\zeta_{X^{\alpha}}=\zeta_{X} / \alpha .
\end{gathered}
$$

48. $L(x)$ is said to be slowly varying (e.g., Embrechts, Kluppelberg, and Mikosch [1997, p. 564]) if for all $t>0, \lim _{x \rightarrow \infty} L(t x) / L(x)=1$. Prototypical examples are $L=a$ and $L(x)=a \ln x$ for a nonzero constant $a$.

49. This makes the use of the kurtosis invalid. As the theoretical kurtosis is infinite, empirical measures of it are essentially meaningless. As a symptom, according to Lévy's theorem, the median sample kurtosis of $T$ i.i.d. demeaned variables $r_{1}, \ldots, r_{T}$, with $\kappa_{T}=\left(\sum_{i=1}^{T} r_{i}^{4} / T\right) /\left(\sum_{i=1}^{T} r_{i}^{2} / T\right)^{2}$, increases to $+\infty$ like $T^{1 / 3}$ if $\zeta_{r}=3$. The use of kurtosis should be banished from use with fat-tailed distributions. As a simple diagnostic for having "fatter tail than from normality," we would recommend, rather than the kurtosis, quantile measures such as $P\left(\left|(r-\bar{r}) / \sigma_{r}\right|>1.96\right) / .05-1$, which is positive if tails are fatter than predicted by a Gaussian. 
For instance, if $X$ is a power-law variable for $\zeta_{X}<\infty$, and $Y$ is power-law variable with an exponent $\zeta_{Y} \geq \zeta_{X}$, or even normal, lognormal, or exponential variable (so that $\zeta_{Y}=\infty$ ), then $X+Y$, $X \cdot Y, \max (X, Y)$ are still power laws with the same exponent $\zeta_{X}$. Hence multiplying by normal variables, adding thin-tailed noise, or summing over i.i.d. variables preserves the exponent. This makes theorizing with power law very streamlined. Also, this gives the empiricist hope that those power laws can be measured, even if the data are noisy: although noise will affect statistics such as variances, it will not affect the power-law exponent. Power-law exponents carry over the "essence" of the phenomenon: smaller order effects do not affect the power-law exponent.

For example, our theory gives a mechanism by which $\zeta_{r}=3$. In reality, we observe $\tilde{r}^{\prime}=\tilde{a} \tilde{r}+\tilde{b}$, where $\tilde{a}$ and $\tilde{b}$ are other random factors not modeled in the theory. We will still have $\zeta_{r^{\prime}}=$ $\zeta_{r}=3$ if $\tilde{a}$ and $\tilde{b}$ have thinner tails than $\tilde{r}\left(\zeta_{a}, \zeta_{b} \geq 3\right)$. If the theory of $\tilde{r}$ captures the first-order effects (those with dominating power law), its predictions for the power-law exponents of the noisy empirical counterpart $\tilde{r}^{\prime}$ will hold.

Proof. See Breiman [1965] and Gnedenko and Kolmogorov [1968] for rigorous proofs, and Sornette [2000] for heuristic derivations. Here we just indicate the proofs for the simplest cases. By induction it is enough to prove the properties for $n=2$ variables:

$$
\begin{aligned}
& \quad P(\max (X, Y)>x)=1-P(\max (X, Y)<x)=1-P(X<x \text { and } \\
& Y<x)=1-P(X<x) P(Y<x)=1-\left(1-\frac{C}{x^{\zeta x}}\right)\left(1-\frac{C^{\prime}}{x^{\zeta y}}\right) \sim \frac{C^{\prime \prime}}{x^{\min (\zeta x, \zeta Y)}}, \\
& \text { where } C^{\prime \prime}=C \text { if } \zeta_{X}<\zeta_{Y}, C^{\prime \prime}=C^{\prime} \text { if } \zeta_{X}>\zeta_{Y} \text {, and } C=C+C^{\prime} \\
& \text { if } \zeta_{X}=\zeta_{Y} \text {. } \\
& \qquad P(\min (X, Y)>x)=P(X>x \text { and } Y>x) \\
& \qquad P(X>x) P(Y>x)=\frac{C C^{\prime}}{x^{\zeta X+\zeta Y}} .
\end{aligned}
$$

Finally, if $P(X>x) \sim C x^{-\zeta_{x}}$, then

$$
P\left(X^{\alpha}>x\right)=P\left(X>x^{1 / \alpha}\right) \sim C\left(x^{1 / \alpha}\right)^{-\zeta x} \sim C x^{-\zeta x / \alpha} .
$$




\section{Estimating Power-Law Exponents}

There are two basic methodologies for estimating powerlaw exponents. We illustrate them with the example of absolute returns. In both methods, one first selects a cutoff of returns, and orders the observations above this cutoff as $r_{(1)} \geq \ldots \geq r_{(n)}$. There is yet no consensus on how to pick the optimal cutoff, as systematic procedures require the econometrician to estimate further parameters [Embrechts, Kluppelberg, and Mikosch 1997]. Often, the most reliable procedure is to use a simple rule, such as choosing all the observations in the top 5 percent.

The first method is a "log rank log size regression," where $\zeta$ is estimated as the OLS coefficient on $r_{(i)}$ in the regression of $\log$ of the rank $i$ on the log size:

$$
\ln i=A-\hat{\zeta}^{O L S} \ln r_{(i)}+\text { noise }
$$

with asymptotic standard error $\hat{\zeta}^{O L S} \cdot(n / 2)^{-1 / 2}$ [Gabaix and Ibragimov 2006]. This method is the simplest, and yields a visual goodness of fit for the power law. This is the approach used, for instance, in Figure I. The second method is Hill's estimator

$$
\hat{\zeta}^{\text {Hill }}=(n-1) / \sum_{i=1}^{n-1}\left(\ln r_{(i)}-\ln r_{(n)}\right),
$$

which has an asymptotic standard error $\hat{\zeta}^{\text {Hill }} n^{-1 / 2}$.

Both methods have pitfalls, discussed in Embrechts, Kluppelberg, and Mikosch [1997, pp. 330-345] and Gabaix and Ioannides [2004]. One large pitfall is the assumption of independent observations. In reality, trading activity is autocorrelated which causes standard errors to be underestimated; however, point estimates remain unbiased. In a future paper we plan to propose a method of estimating the standard errors. In any case, the stability of the estimates across different periods, countries, and classes of assets gives us confidence that the empirical estimates we report here are robust.

With the samples of millions of points available in finance, standard errors are so small that one can reject essentially any null hypothesis. Hence, researchers estimating power laws typically do not use tests to see whether a distribution with more parameters would offer a better fit. With so many data points, statistical tests would always justify a higher-dimen- 
sional parameterization, even though economically, the improvement in fit would be minimal. Rather, $\hat{\zeta}$ is best interpreted as the optimal one-parameter approximation of the tail by a Pareto family. Explaining the value of this one-parameter approximation is already a difficult challenge. Explaining the higher order terms may be best left for future decades of research.

\section{APPEndix 2: Proofs}

Proof of Proposition 2. We use $T=V / \bar{V}$ and $B(1)=0$ to calculate

$$
\begin{aligned}
& \operatorname{var}\left[\int_{1}^{1+T} B(t) d t\right]=\operatorname{var}\left[\int_{0}^{T}\left(\int_{0}^{s} d B(1+u)\right) d s\right] \\
&=\operatorname{var}\left[\int_{0}^{T}\left(\int_{u}^{T} d s\right) d B(u+1)\right]=\operatorname{var}\left[\int_{0}^{T}(T-u) d B(u+1)\right] \\
&=\int_{0}^{T}(T-u)^{2} d u=\frac{T^{3}}{3}=\frac{V^{3}}{3 \bar{V}^{3}} .
\end{aligned}
$$

The liquidity provider sells $V$ shares to the fund at a price $p+\pi+\tau$, and replenishes her inventory during $[1,1+T]$ at a total cost $K=\int_{1}^{1+T} p(t) \bar{V} d t$. Her net income from the transaction is

$$
\begin{aligned}
W=(p+\pi+\tau) V-\int_{1}^{1+T} p(t) \bar{V} d t=(p+\pi+\tau) V \\
\quad-\int_{1}^{1+T}(p+\pi+\sigma B(t)) \bar{V} d t=\tau V-\sigma \bar{V} \int_{1}^{1+T} B(t) d t .
\end{aligned}
$$

Her utility is

$$
\begin{array}{r}
U=E[W]-\lambda(\operatorname{var} W)^{\delta / 2}=\tau V-\lambda\left(\sigma^{2} \bar{V}^{2} \operatorname{var}\left[\int_{1}^{1+T} B(t) d t\right]\right)^{\delta / 2} \\
=\tau V-\lambda\left(\frac{\sigma^{2} V^{3}}{3 \bar{V}}\right)^{\delta / 2} .
\end{array}
$$


The fund has full bargaining power, and so leaves the liquidity supplier with a reservation utility $U=0$. This implies that

$$
\tau=\lambda\left(\frac{\sigma^{2}}{3 \bar{V}}\right)^{8 / 2} V^{38 / 2-1} .
$$

Economically, the liquidity provider purchases the stock back at an average price $\bar{p}=T^{-1} \int_{1}^{1+T} p(t) d t$, which has expected value $p+\pi$ and standard deviation $\sigma(V / 3 \bar{V})^{1 / 2}$. The temporary impact $\tau$ is the compensation for this price risk of $\sigma(V / 3 \bar{V})^{1 / 2}$.

Proof of Proposition 4. In this proof we use the notation $V(M)$ rather than $V(M, S)$. The Lagrangian is

$$
\begin{aligned}
\mathscr{C}=\int V(M)(M-R(V(M))) f(M) d M-\mu \int V(M) R(V(M)) \\
\times f(M) d M=\int V(M)\left(M-(1+\mu) h V(M)^{\gamma}\right) f(M) d M .
\end{aligned}
$$

It is sufficient to optimize on $V(M)$ separately for each $M$ :

$$
\begin{aligned}
& \text { (45) } 0=\frac{\partial \mathscr{C}}{\partial V(M)}=\frac{\partial}{\partial V(M)}\left[V(M) M-(1+\mu) h V(M)^{1+\gamma}\right] f(M) \rightarrow 0 \\
& =M-(1+\mu)(1+\gamma) h V(M)^{\gamma} \rightarrow V(M)=[(1+\mu)(1+\gamma) h]^{-1 / \gamma} M^{1 / \gamma} .
\end{aligned}
$$

Thus, using equation (17),

$$
\begin{aligned}
-E\left[r_{t} \mid \tilde{C}=0\right] & =E\left[h V(M)^{1+\gamma} / S\right] \\
& =h E\left[M^{1+1 / \gamma}\right][(1+\mu)(1+\gamma) h]^{-(1+1 / \gamma)} / S .
\end{aligned}
$$

Constraint (18) binds if and only if $\mu>0$, i.e.,

$$
S<S^{*}
$$

with

$$
S^{*}=\frac{E\left[M^{1+1 / \gamma}\right][(1+\gamma)]^{-(1+1 / \gamma)}}{h^{1 / \gamma} \Lambda} .
$$

If the constraint binds, $-E\left[r_{t} \mid \tilde{C}=0\right]=\Lambda$. This implies that

$$
[(1+\mu)(1+\gamma) h]^{-(1+1 / \gamma)}=\frac{\Lambda S}{h E\left[M^{1+1 / \gamma}\right]}
$$


and going back to equation (45), we get $V(M)=v M^{1 / \gamma} S^{1 /(1+\gamma)}$ with

$$
v=\left(\frac{\Lambda}{h E\left[M^{1+1 / \gamma}\right]}\right)^{1 /(1+\gamma)} .
$$

The expression for $R$ comes from $R=h V^{\gamma}$.

To calibrate $S^{*}$, we use the following parameters, which we view as simply indicative: $\gamma=1 / 2, E\left[M^{3}\right]^{1 / 3}=10$ percent (which is less than the annual standard deviation of the market, hence likely to be conservative), $\Lambda=2$ percent of price impact costs paid annually. ${ }^{50}$ We take a price impact, motivated by subsections II.C and III.A: $R(V)=A \sigma(V / D)^{1 / 2}$, where $\sigma=$ daily market volatility $=0.01, A=1 / 2$, which means that up to $A^{2}=25$ percent of the market fluctuations are due to our effects, $D=$ daily market turnover. Using the 1999 number of a total equity market capitalization of $\$ 18$ trillion, and a 50 percent annual turnover, $D=$ $1 / 2 \times \$ 18$ trillion $/ 250=\$ 36$ billion. So

$$
S^{*}=\frac{D}{A^{2}(3 / 2)^{3}} \frac{E\left[M^{3}\right]}{\sigma^{2} \Lambda}=\$ 21 \text { trillion. }
$$

Proof of Proposition 5. We start from equation (21). We apply the rules in Appendix 1 to derive

$$
\begin{aligned}
\zeta_{R} & =\zeta_{h v^{\vee M S} S^{\gamma /(1+\gamma)}}=\zeta_{M S \gamma /(1+\gamma)} \text { by applying }(41) \\
& =\min \left[\zeta_{M}, \zeta_{S \gamma^{\gamma /(1+\gamma)}}\right] \text { by applying }(38) \\
& =\min \left[\zeta_{M}, \frac{1+\gamma}{\gamma} \zeta_{S}\right] \text { by applying (42), }
\end{aligned}
$$

which proves the proposition. One derives $\zeta_{V}$ in the same way.

Proof of Proposition 6. Assumption 2 implies that $\zeta_{M} \geq 1+$ $1 / \gamma=3$. Then, Proposition 5 gives $\zeta_{R}=\min \left(3, \zeta_{M}\right)=3$ and $\zeta_{V}=$ $\gamma \zeta_{R}=3 / 2$.

Proof of Proposition 7. We will start with the following lemma, which means that if $X$ has fatter tails than $Y$, then $E[X \mid X Y=z]$ is proportional to $z$ for large $z$. The reason is that an extreme value of $X Y$ probably comes from an extreme value of $X$. 50. If the fund gets $F$ signals per year, $S^{*}$ is divided by $F^{1 / 2}$, as $M$ is divided
by $F^{1 / 2}$ and $\Lambda$ by $F$. 
Lemma 8. Suppose that $X$ and $Y$ are independent random variables, with exact power distributions: $P(X>x)=\left(x / x^{*}\right)^{-\zeta_{x}}$, $P(Y>y)=\left(y / y^{*}\right)^{-\zeta_{Y}}$ for $x \geq x^{*}$ and $y \geq y^{*}$. Define $z^{*}=$ $x^{*} y^{*}$. Assume that $\zeta_{X} \leq \zeta_{Y}$. Then,

$$
\begin{aligned}
& E[X \mid X Y=z]=L(z) z \\
& L(z)=\frac{E\left[Y^{\zeta X-1} 1_{Y<z / x^{*}}\right]}{E\left[Y^{\zeta x} 1_{Y<z / x^{*}}\right]} .
\end{aligned}
$$

$L(z)$ is a slowly varying function. If $\zeta_{X}<\zeta_{Y}$,

$$
\lim _{z \rightarrow \infty} L(z)=\frac{E\left[Y^{\zeta x-1}\right]}{E\left[Y^{\zeta x}\right]} .
$$

If $\zeta_{X}=\zeta_{Y}$,

$$
L(z)=\frac{1}{y^{*}} \frac{1-z^{*} / z}{\ln \left(z / z^{*}\right)} \sim \frac{1}{y^{*}} \frac{1}{\ln z} \text { for } z \rightarrow \infty .
$$

Proof of Lemma 8. By normalization, it is enough to study the case $x^{*}=y^{*}=1$, calling $f$ and $g$ the densities of $X$ and $Y$. By Bayes' rule, $p(X=x \mid X Y=z)=k f(x) g(z / x) / x$ for a constant $k$. So,

$$
E[X \mid X Y=z]=\frac{\int x f(x) g(z / x) / x d x}{\int f(x) g(z / x) / x d x}=z \frac{\int f(z / y) g(y) / y^{2} d y}{\int f(z / y) g(y) / y^{1} d y}
$$

(by the change of variable $x=z / y)=z \frac{\int_{1}^{z}(z / y)^{-\zeta x^{-1}} g(y) / y^{2} d y}{\int_{1}^{z}(z / y)^{-\zeta x^{-1}} g(y) / y d y}$

$$
=z \frac{\int_{1}^{z} y^{\zeta X-1} g(y) d y}{\int_{1}^{z} y^{\zeta x} g(y) d y}=z \frac{E\left[Y^{\zeta x-1} 1_{Y<z}\right]}{E\left[Y^{\zeta x} 1_{Y<z}\right]}=z L(z) .
$$

When $\zeta_{X}<\zeta_{Y}, E\left[Y^{\zeta_{X}} 1_{Y<z}\right] \rightarrow E\left[Y^{\zeta_{X}}\right]<\infty$.

When $\zeta_{X}=\zeta_{Y}$,

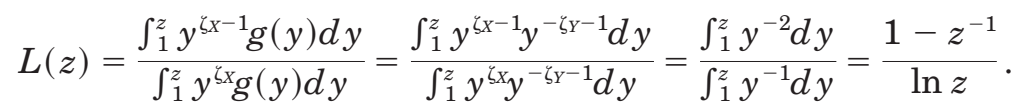

For the proof of Proposition 7, we use Lemma 8 with $X=M$ and $Y=h v^{\gamma} S^{\gamma /(1+\gamma)}$. We call $\zeta_{M}^{\text {subj }}$ the exponent of the distribution agents use when they calculate the conditional expectation (28). Given the hypothesis $\zeta_{M}^{s u b j} \leq \zeta_{R}$, equation (23) gives $\zeta_{M}^{s u b j} \leq$ $\zeta_{R} \leq(1+1 / \gamma) \zeta_{S}=\zeta_{Y}$. So using Lemma 8, 


$$
E\left[M \mid R=h v^{\gamma} M S^{\gamma /(1+\gamma)}=X Y\right]=R L(R)
$$

for a slowly varying function $L(R)$ of $R$. In the case $\zeta_{M}^{\text {subj }}<(1+$ $1 / \gamma) \zeta_{S}$, we get $\lim _{R \rightarrow \infty} L(R)=b^{\prime}=E\left[Y^{s_{M}^{s u b j}}-1\right] / E\left[Y^{s_{M}^{s u b j}}\right]$, a constant. Finally, given $\pi=R L(R)$, and $L$ is slowly varying, $\zeta_{\pi}=\zeta_{R}$.

\section{Appendix 3: Confidence Intervals and Tests When a Variable Has InFInite Variance}

\section{A. Construction of the Confidence Intervals for Figure VI}

In a given bin conditioned by $Q=Q_{i}$, with $k$ elements $r_{1}^{2}, \ldots, r_{k}^{2}$, the point estimate of $E\left[r^{2} \mid Q=Q_{i}\right]$ is the sample mean of the $r_{j}^{2}$, which we call $m$. Getting a confidence interval for $m$ is delicate, as $r^{2}$ has infinite variance, so the standard approach relying on asymptotic normality is invalid. But the theory of self-normalizing sums of Logan, Mallows, Rice, and Shepp [1973] shows that if $\mu$ is the true mean and $\sigma$ is empirical standard deviation of the $r_{j}^{2}$ in the bin with $k$ observations, then the ratio $t=k^{1 / 2}(m-\mu) / \sigma$ follows a nondegenerate distribution for large $k$. By Monte Carlo analysis we simulate draws following a power law with exponent 1.5, which is the exponent of $r^{2}$, and we tabulate 2.5 percent and 97.5 percent quantiles of $-t$, which we call $-\chi^{-}=-1.1$ and $\chi^{+}=5.5$. They differ from their finite variance value, which would be $\chi^{-}=\chi^{+}=1.95 .^{51}$

To construct 95 percent confidence intervals, we can first calculate the empirical standard error $\Delta r_{i}^{2}=\sigma_{r_{i, j}^{2}} k^{-1 / 2}$, the sample standard deviation of the observations divided by the square root of the number of observations. A 95 percent confidence interval is $\left[m_{i}-\chi^{-} \Delta r_{i}^{2}, m_{i}+\chi^{+} \Delta r_{i}^{2}\right]$. We should stress that we make the simplifying assumption of independent and identically distributed draws. Given that the data are likely to be autocorrelated, our confidence intervals are likely to be too narrow.

B. Test of Relation (7): $E\left[r^{2} \mid Q\right]=\alpha+\beta Q$

For each bin $Q_{i}$ of $Q$, we set $r_{i}^{2}=E\left[r^{2} \mid Q=Q_{i}\right]$, and $\Delta r_{i}^{2}$ sample standard error in interval $i$. By least squares we fit an affine relationship $E\left[r^{2} \mid Q\right]=g(Q)$, with

51. When $k$ is finite, there is some sensitivity of $\chi^{-}$and $\chi^{+}$to the underlying distribution. We take a pure power law $P\left(r^{2}>x\right)=x^{-3 / 2}$ for $x \geq 1$, and $k=200$, to reflect our typical sample size in bins of extreme values. 


$$
\begin{aligned}
& g(Q)=0.07+0.60 Q \\
& \text { (0.59) (0.013). }
\end{aligned}
$$

The standard errors are in parentheses, and the $R^{2}=0.90$. We find that for all values $Q_{i} \geq 3$, the predicted value $g\left(Q_{i}\right)$ belongs to the 95 percent confidence interval: $g\left(Q_{i}\right) \in\left[r_{i}^{2}-\chi^{-} \Delta r_{i}^{2}, r_{i}^{2}+\right.$ $\left.\chi^{+} \Delta r_{i}^{2}\right]$. We conclude that, at the 95 percent confidence level, we cannot reject the linear form $E\left[r^{2} \mid Q\right]=g(Q)$ for $Q \geq 3$.

Massachusetts Institute of Technology, Department of Economics, and National Bureau of Economic RESEARCH

Boston University, Department of Physics, Center for Polymer Studies Boston University, Department of Physics, Center for Polymer Studies Boston University, Department of Physics, Center for Polymer Studies

\section{REFERENCES}

Abreu, Dilip, and Markus Brunnermeier, "Bubbles and Crashes," Econometrica, LXXI (2003), 173-204.

Acharya, Viral, and Lasse Pedersen, "Asset Pricing with Liquidity Risk," Journal of Financial Economics, LXV (2005), 375-410.

Almgren, Robert, and Neil Chriss, "Optimal Execution of Portfolio Transactions," Journal of Risk, III (2000), 5-39.

Andersen, Torben G., Tim Bollerslev, Francis Diebold, and Heiko Ebens, "The Distribution of Realized Stock Return Volatility," Journal of Financial Economics, LXI (2001), 43-76.

Arthur, W. Brian, Blake LeBaron, John H. Holland, Richard Palmer, and Paul Tayler, "Asset Pricing under Endogenous Expectations in an Artificial Stock Market," in W. Brian Arthur, Steven Durlauf, and David Lane, eds., The Economy as an Evolving Complex System II (Reading, MA: Addison-Wesley, 1997).

Axtell, Robert, "Zipf Distribution of U. S. Firm Sizes," Science, CCXCIII (2001), $1818-1820$.

Backus, David, Bryan R. Routledge, and Stanley E. Zin, "Exotic Preferences for Macroeconomists," NBER Macroeconomics Annual (Cambridge, MA: MIT Press, 2005).

Bak, Per, Ken Chen, José Scheinkman, and Michael Woodford, "Aggregate Fluctuations from Independent Sectoral Shocks: Self-Organized Criticality in a Model of Production and Inventory Dynamics," Ricerche Economiche, XLVII (1993), 3-30.

Barberis, Nicholas, Ming Huang, and Tano Santos, "Prospect Theory and Asset Prices," Quarterly Journal of Economics, CXVI (2001), 1-53.

Barberis, Nicholas, and Richard Thaler, "A' Survey of Behavioral Finance," in George Constantinides and René Stulz, eds., Handbook of the Economics of Finance (Amsterdam: North-Holland, 2003).

Barclay, Michael, and Jerold Warner, "Stealth Trading and Volatility Which Trades Move Prices?" Journal of Financial Economics, XXXIV (1993), 281-305.

Barra, Market Impact Model Handbook (Berkeley, CA: Barra, 1997).

Barro, Robert, "Rare Disasters and Asset Markets in the Twentieth Century," Quarterly Journal of Economics, CXXI (2006), forthcoming.

Bernardo, Antonio, and Ivo Welch, "Liquidity and Financial Market Runs," Quarterly Journal of Economics, CXIX (2004), 135-158.

Bertsimas, Dimitris, and Andrew Lo, "Optimal Control of Execution Costs," Journal of Financial Markets, I (1998), 1-50.

Biais, Bruno, Lawrence Glosten, and Chester Spatt, "Market Microstructure: A 
Survey of Microfoundations, Empirical Results, and Policy Implications," Journal of Financial Markets, VIII (2005), 217-264.

Blume, Lawrence, and Stephen Durlauf, eds., The Economy as an Evolving Complex System III (New York: Oxford University Press, 2005).

Bouchaud, Jean-Philippe, and Marc Potters, Theory of Financial Risks and Derivative Pricing: From Statistical Physics to Risk Management, second edition (New York: Cambridge University Press, 2003).

Bouchaud, Jean-Philippe, Yuval Gefen, Marc Potters, and Matthieu Wyart, "Fluctuations and Response in Financial Markets: The Subtle Nature of 'Random' Price Changes," Quantitative Finance, IV (2004), 176-190.

Brady, Nicholas, and United States' Presidential Task Force on Market Mechanisms, Report of the Presidential Task Force on Market Mechanisms Submitted to the President of the United States (Washington, DC: Docs., U. S. G.P.O., 1988).

Breiman, Leonard, "On Some Limit Theorems Similar to the Arc-Sin Law," Theory of Probability and its Applications, X (1965), 323-330.

Brock, William, and Cars Hommes, "Rational Routes to Randomness," Econometrica, LXV (1998), 1059-1096.

Brunnermeier, Markus, and Stefan Nagel, "Hedge Funds and the Technology Bubble," Journal of Finance, LIX (2004), 2013-2040.

Campbell, John, Tarun Ramadorai, and Tuomo Vuolteenaho, "Caught on Tape: Institutional Order Flow and Stock Returns," National Bureau of Economic Research Working Paper No. 11439, 2005.

Campbell, John, and Robert Shiller, "The Dividend-Price Ratio and Expectations of Future Dividends and Discount Factors," Review of Financial Studies, I (1988), 195-228.

Canals, Claudia, Xavier Gabaix, Josep Vilarrubia, and David Weinstein, "Trade Patterns, Trade Balances, and Idiosyncratic Shocks," Working Paper, Columbia University and Massachusetts Institute of Technology, 2005.

Chae, Joon, and Jonathan Lewellen, "Herding, Feedback Trading, and Stock Returns: Evidence from Korea," Working Paper, Dartmouth College, 2005.

Chan, Louis, and Josef Lakonishok, "Institutional Trades and Intraday Stock Price Behavior," Journal of Financial Economics, XXXIII (1993), 173-189.

Chan, Louis, and Josef Lakonishok, "The Behavior of Stock Prices around Institutional Trades," Journal of Finance, L (1995), 1147-1174.

Chiyachantana, Chiraphol, Pankaj Jain, Christine Jiang, and Robert Wood, "International Evidence on Institutional Trading Behavior and Price Impact," Journal of Finance, LIX (2004), 869-898.

Choe, Hyuk, Bong-Chan Kho, and René Stulz, "Do Foreign Investors Destabilize Stock Markets? The Korean Experience in 1997," Journal of Financial Economics, LIV (1999), 227-264.

Clark, Peter, "A Subordinated Stochastic Process Model with Finite Variance for Speculative Prices," Econometrica, XLI (1973), 135-155.

Cohen, Randolph, Paul Gompers, and Tuomo Vuolteenaho, "Who Underreacts to Cash-Flow News? Evidence from Trading between Individuals and Institutions," Journal of Financial Economics, LXVI (2002), 409-462.

Corsetti, Giancarlo, Paolo Pesenti, and Nouriel Roubini, "The Role of Large Players in Currency Crises," in Sebastian Edwards and Jeffrey Frankel, eds., Preventing Currency Crises in Emerging Markets (Chicago, IL: University of Chicago Press and NBER, 2002).

Coyne, Kevin, and Jonathan Witter, "Taking the Mystery out of Investor Behavior," Harvard Business Review, LXXX (2002), 68-79.

Cutler, David, James Poterba, and Lawrence Summers, "What Moves Stock Prices?" Journal of Portfolio Management, XV (1989), 4-12.

Davies, James, and Anthony Shorrocks, "The Distribution of Wealth," in Anthony Atkinson and Francois Bourguignon, eds., Handbook of Income Distribution, Volume I (Amsterdam; New York; and Oxford: Elsevier Science, North-Holland, 2000).

Dembo, Amir, Jean-Dominique Deuschel, and Darrell Duffie, "Large Portfolio Losses," Finance and Stochastics, VIII (2004), 3-16.

Durlauf, Steven, "Non-Ergodic Economic Growth," Review of Economic Studies, LX (1993), 349-366. 
Easley, David, and Maureen O'Hara, "Price, Trade Size, and Information in Securities Markets," Journal of Financial Economics, XIX (1987), 69-90.

Embrechts, Paul, Claudia Kluppelberg, and Thomas Mikosch, Modelling Extremal Events (New York: Springer Verlag, 1997).

Engle, Robert, and Jeffrey Russell, "Autoregressive Conditional Duration: A New Model for Irregularly Spaced Transaction Data," Econometrica, LXVI (1998), $1127-1162$.

Epstein, Lawrence, and Stanley Zin, “'First-Order' Risk Aversion and the Equity Premium Puzzle," Journal of Monetary Economics, XXVI (1990), 387-407.

Evans, Martin, and Richard Lyons, "Order Flow and Exchange Rate Dynamics," Journal of Political Economy, CX (2002), 170-180.

Fair, Ray, "Events that Shook the Market," Journal of Business, LXXV (2002), 713-731.

Fama, Eugene, "Mandelbrot and the Stable Paretian Hypothesis," Journal of Business, XXXVI (1963), 420-429.

Farmer, Doyne, and Fabrizio Lillo, "On the Origin of Power-Law Tails in Price Fluctuations," Quantitative Finance, IV (2004), C7-11.

French, Kenneth, and Richard Roll, "Stock Return Variance: The Arrival of Information and the Reaction of Traders," Journal of Financial Economics, XVII (1986), 99-117.

Froot, Kenneth A., Paul O. Connell, and Mark Seasholes, "The Portfolio Flows of International Investors," Journal of Financial Economics, LIX (2001), 151-193.

Fujiwara, Yoshi, Corrado Di Guilmi, Hideaki Aoyama, Mauro Gallegati, and Wataru Souma, "Do Pareto-Zipf and Gibrat Laws Hold True? An Analysis with European Firms," Physica A, CCCXXXV (2004), 197-216.

Gabaix, Xavier, "Zipf's Law for Cities: An Explanation," Quarterly Journal of Economics, CXIV (1999), 739-767.

_ , "The Granular Origins of Aggregate Fluctuations," Working Paper, Massachusetts Institute of Technology, 2005.

Gabaix, Xavier, Parameswaran Gopikrishnan, Vasiliki Plerou, and H. Eugene Stanley, "A Theory of Power Law Distributions in Financial Market Fluctuations," Nature, CDXXIII (2003), 267-230.

Gabaix, Xavier, Parameswaran Gopikrishnan, Vasiliki Plerou, and H. Eugene Stanley, "Are Stock Market Crashes Outliers?" Working Paper, Massachusetts Institute of Technology, 2005.

Gabaix, Xavier, and Rustam Ibragimov, "Rank-1/2: A Simple Way to Improve the OLS Estimation of Tail Exponents," Working Paper, Harvard University, 2006.

Gabaix, Xavier, and Yannis Ioannides, "The Evolution of the City Size Distributions," in J. Vernon Henderson and Jacques-François Thisse, eds., Handbook of Regional and Urban Economics, Volume IV (Amsterdam: Elsevier NorthHolland, 2004).

Gabaix, Xavier, Rita Ramalho, and Jonathan Reuter, "Investor Behavior and Mutual Fund Dynamics," Working Paper, Massachusetts Institute of Technology, 2005.

Gennotte, Gerard, and Hayne Leland, "Market Liquidity, Hedging, and Crashes," American Economic Review, LXXX (1990), 999-1021.

Gilboa, Itzhak, and David Schmeidler, "Maxmin Expected Utility with NonUnique Prior," Journal of Mathematical Economics, XVIII (1989), 141-153.

Gnedenko, Boris V., and Andrej N. Kolmogorov, Limit Distributions for Sums of Independent Random Variables (Reading, MA: Addison-Wesley, 1968).

Goetzmann, William, and Massimo Massa, "Index Funds and Stock Market Growth," Journal of Business, LXXVI (2003), 1-28.

Gompers, Paul, and Andrew Metrick, "Institutional Investors and Equity Prices," Quarterly Journal of Economics, CXVI (2001), 229-259.

Gopikrishnan, Parameswaran, Vasiliki Plerou, Luis Amaral, Martin Meyer, and H. Eugene Stanley, "Scaling of the Distribution of Fluctuations of Financial Market Indices," Physical Review E, LX (1999), 5305-5316.

Gopikrishnan, Parameswaran, Vasiliki Plerou, Xavier Gabaix, and H. Eugene Stanley, "Statistical Properties of Share Volume Traded in Financial Markets," Physical Review E, LXII (2000), R4493-R4496. 
Greenwald, Bruce, and Jeremy Stein, "Transactional Risk, Market Crashes, and the Role of Circuit Breakers," Journal of Business, LXIV (1991), 443-462.

Griffin, John, Jeffrey Harris, and Selim Topaloglu, "The Dynamics of Institutional and Individual Trading," Journal of Finance, LVIII (2003), 2285-2320.

Grinold, Richard C., and Ronald N. Kahn, Active Portfolio Management (Boston, MA; London: Irwin/McGraw-Hill, 1999).

Grossman, Sanford, and Merton Miller, "Liquidity and Market Structure," Journal of Finance, XLIII (1988), 617-633.

Guillaume, Dominique M., Michel M. Dacorogna, Rakhal R. Davé, Ulrich A. Müller, Richard B. Olsen, and Olivier V. Pictet, "From the Bird's Eye to the Microscope: A Survey of New Stylized Facts of the Intra-Daily Foreign Exchange Markets," Finance and Stochastics, I (1997), 95-129.

Gul, Faruk, "A Theory of Disappointment Aversion," Econometrica, LIX (1991), $667-686$.

Hansen, Lars, and Thomas Sargent, "Robustness," manuscript, University of Chicago and New York University, 2005.

Hasbrouck, Joel, "Measuring the Information-Content of Stock Trades," Journal of Finance, XLVI (1991), 179-207.

Hasbrouck, Joel, and Duane Seppi, "Common Factors in Prices, Order Flows and Liquidity," Journal of Financial Economics, LIX (2001), 388-411.

Hirshleifer, David, "Investor Psychology and Asset Pricing," Journal of Finance, LVI (2001), 1533-1598.

Huberman, Gur, and Werner Stanzl, "Price Manipulation and Quasi-Arbitrage," Econometrica, LXXIV (2004), 1247-1276.

Hvidkjaer, Soeren, "A Trade-based Analysis of Momentum," Review of Financial Studies, XVIII (2005),

Ibragimov, Rustam, "Portfolio Diversification and Value at Risk under ThickTailedness," Working Paper, Harvard University, 2005.

Jackson, Matthew, and Brian Rogers, "Search in the Formation of Large Networks: How Random Are Socially Generated Networks?" Working Paper, California Institute of Technology, 2005.

Jansen, Dennis, and Casper de Vries, "On the Frequency of Large Stock Returns: Putting Booms and Busts into Perspective," Review of Economics and Statistics, LXXIII (1991), 18-24.

Jones, Charles M., and Marc Lipson, "Sixteenths: Direct Evidence on Institutional Execution Costs," Journal of Financial Economics, LIX (2001), 253-278.

Kahneman, Daniel, and Amos Tversky, "Prospect Theory: An Analysis of Decision under Risk," Econometrica, XLVII (1979), 263-291.

Keim, Donald, and Ananth Madhavan, "The Upstairs Market for Large-Block Trades: Analysis and Measurement of Price Effects," Review of Financial Studies, IX (1996), 1-36.

Kou, Samuel, and Steven Kou, "A Diffusion Model for Growth Stocks," Mathematics of Operations Research, XXXIX (2004), 191-212.

Kyle, Albert, "Continuous Auctions and Insider Trading," Econometrica, LIII (1985), 1315-1335.

LeRoy, Stephen, and Richard Porter, "The Present-Value Relation: Tests Based on Implied Variance Bounds," Econometrica, XLIX (1981), 555-574.

Levy, Moshe, Heim Levy, and Sorin Solomon, Microscopic Simulation of Financial Markets: From Investor Behavior to Market Phenomena (San Diego, CA: Academic Press, 2000).

Liesenfeld, Roman, "A Generalized Bivariate Mixture Model for Stock Price Volatility and Trading Volume," Journal of Econometrics, CIV (2001), 141-178.

Lillo, Fabrizio, and Doyne Farmer, "The Long Memory of the Efficient Market," Studies in Nonlinear Dynamics \& Econometrics, VIII (2004), Article 1, 1-33.

Liu, Yanhui, Parameswaran Gopikrishnan, Pierre Cizeau, Martin Meyer, ChungKang Peng, and H. Eugene Stanley, "Statistical Properties of the Volatility of Price Fluctuations," Physical Review E, LX (1999), 1390-1400.

Lo, Andrew, and Jiang Wang, "Trading Volume: Definitions, Data Analysis, and Implications of Portfolio Theory," Review of Financial Studies, XIII (2001), $257-300$.

Loeb, Thomas, "Trading Cost: The Critical Link between Investment Information and Results," Financial Analysts Journal, XXXIX (1983), 39-44. 
Logan, Benjamin F., Colin Mallows, Stephen Rice, and Larry Shepp, "Limit Distributions of Self-Normalized Sums," Annals of Probability, I (1973), $788-809$.

Lux, Thomas, "The Stable Paretian Hypothesis and the Frequency of Large Returns: An Examination of Major German Stocks," Applied Financial Economics, VI (1996), 463-475.

Lux, Thomas, and Didier Sornette, "On Rational Bubbles and Fat Tails," Journal of Money, Credit and Banking, XXXIV (2002), 589-610.

Mandelbrot, Benoit, "The Variation of Certain Speculative Prices," Journal of Business, XXXVI (1963), 394-419.

Mantegna, Rosario, and H. Eugene Stanley, "Scaling Behavior in the Dynamics of an Economic Index," Nature, CCCVXXVI (1995), 46-49.

Mantegna, Rosario, and H. Eugene Stanley, An Introduction to Econophysics: Correlations and Complexity in Finance (Cambridge, UK: Cambridge University Press, 2000).

Marsh, Terrance, and Niklas Wagner, "Return-Volume Dependence and Extremes in International Equity Markets," Working Paper, University of California at Berkeley, 2004.

Maslov, Sergei, and Mark Mills, "Price Fluctuations from the Order Book Perspective: Empirical Facts and a Simple Model," Physica A, CCXCIX (2001), 234-246.

McCulloch, J. Houston, "Financial Applications of Stable Distributions," in G. S. Maddala and C. R. Rao, eds., Statistical Methods in Finance (Amsterdam: Elsevier North-Holland, 1996).

O'Hara, Maureen, Market Microstructure Theory (Cambridge, MA: Blackwell, 1995).

Okuyama, Kazumi, Misako Takayasu, and Hideki Takayasu, "Zipf's Law in Income Distribution of Companies," Phvsica A. CCLXIX (1999), 125-131.

Piketty, Thomas, and Emmanuel Saez, "Income Inequality in the United States, 1913-1998," Quarterly Journal of Economics, CXVIII (2003), 1-39.

Plerou, Vasiliki, Parameswaran Gopikrishnan, Luis Amaral, Martin Meyer, and H. Eugene Stanley, "Scaling of the Distribution of Fluctuations of Financial Market Indices," Physical Review E, LX (1999), 6519-6529.

Plerou, Vasiliki, Parameswaran Gopikrishnan, Luis Amaral, Xavier Gabaix, and H. Eugene Stanley, "Economic Fluctuations and Anomalous Diffusion," Physical Review E, LXII (2000), R3023-R3026.

Plerou, Vasiliki, Parameswaran Gopikrishnan, Xavier Gabaix, and H. Eugene Stanley, "Quantifying Stock Price Response to Demand Fluctuations," Phvsical Review E, LXVI (2002), 027104-1-027104-4.

Plerou, Vasiliki, Parameswaran Gopikrishnan, Xavier Gabaix, and H. Eugene Stanley, "On the Origins of Power-Law Fluctuations in Stock Prices," Quantitative Finance, IV (2004), C11-C15.

Pritsker, Matthew, "Large Investors: Implications for Equilibrium Asset Returns, Shock Absorption and Liquidity," working paper, Federal Reserve Board, 2005.

Pushkin, Dmitri, and Hassan Aref, "Bank Mergers as Scale-Free Coagulation," Physica A, CCCXXXVI (2004), 571-584.

Rachev, Svetlozar, and Stefan Mittnik, Stable Paretian Models in Finance (New York: Wiley, 2000).

Resnick, Sidney, Extreme Values, Regular Variation, and Point Processes (New York: Springer Verlag, 1987).

Rietz, Thomas, "The Equity Risk Premium: A Solution," Journal of Monetary Economics, XXII (1988), 117-131.

Roll, Richard, " $R^{2}$," Journal of Finance, XLIII (1988), 541-566.

Romer, David, "Rational Asset-Price Movements without News," American Economic Review, LXXXIII (1993), 1112-1130.

Routledge, Bryan, and Stanley Zin, "Generalized Disappointment Aversion and Asset Prices," Working Paper, Carnegie Mellon University, 2004.

Seppi, Duane, "Equilibrium Block Trading and Asymmetric Information," Journal of Finance, XLV (1990), 73-94.

Shiller, Robert, "Do Stock Prices Move Too Much to Be Justified by Subsequent Changes in Dividends?" American Economic Review, LXXI (1981), 421-436. 
Shleifer, Andrei, “Do Demand Curves for Stocks Slope Down?" Journal of Finance, XLI (1986), 579-590.

Inefficient Markets: An Introduction to Behavioral Finance (Oxford; New York: Oxford University Press, 2000).

Shleifer, Andrei, and Robert Vishny, "The Limits of Arbitrage," Journal of Finance, LII (1997), 35-55.

Simon, Herbert, "On a Class of Skew Distribution Functions," Biometrika, XLIV (1955), 425-440.

Solomon, Solomon, and Peter Richmond, "Power Laws of Wealth, Market Order Volumes and Market Returns," Physica A, CCXCIX (2001), 188-197.

Sornette, Didier, Critical Phenomena in Natural Sciences (Heidelberg and New York: Springer, 2000).

Vayanos, Dimitri, "Strategic Trading in a Dynamic Noisy Market," Journal of Finance, LV (2001), 131-171.

Weitzman, Martin, "A Unified Bayesian Theory of Equity 'Puzzles'," Working Paper, Harvard University, 2005.

Werner, Ingrid, "NYSE Order Flow, Spreads, and Information," Journal of Financial Markets, VI (2003), 309-335.

Wurgler, Jeffrey, and Ekaterina Zhuravskaya, "Does Arbitrage Flatten Demand Curves for Stocks?" Journal of Business, LXXV (2002), 583-608.

Zhang, Yi-Cheng, "Toward a Theory of Marginally Efficient Markets," Physica A, CCLXIX (1999), 30-44.

Zipf, George, Human Behavior and the Principle of Least Effort (Cambridge, MA: Addison-Wesley, 1949). 